\title{
Design, Synthesis, and Reactivity of 1-Hydrazinodienes For Use in Organic Synthesis
}

\author{
Glenn M. Sammis, Eric M. Flamme, Hao Xie, Douglas M. Ho, and Erik J. Sorensen* \\ Department of Chemistry \\ Princeton University \\ Princeton, New Jersey, 08544-1009
}

\begin{abstract}
General: Scandium(III) triflate and tris(dibenzylideneacetone)dipalladium chloroform adduct were purchased from Strem Chemical. Unless otherwise stated, all reagents were purchased from Aldrich and used without further purification. Methylene chloride, tetrahydrofuran, toluene, and pyridine were dried by passing through activated alumina columns. Reactions were monitored by thin-layer chromatography (TLC) carried out on 0.25 mm Merck silica gel plated (60 $\left.\mathrm{F}_{254}\right)$ using UV light as a visualizing agent and aqueous potassium permanganate and heat as a developing agent. E. Merck silica gel 60 (230-400 mesh) was sued for flash column chromatography.
\end{abstract}

Instrumentation: FT-IR spectra were obtained on a Perkin-Elmer Parargon 500. Nuclear magnetic resonance (NMR) spectra were taken on a Varian Inova-500, Inova-400, and Mercury-Vx-300 instruments. Proton chemical shifts are reported in parts per million and are calibrated to the residual protium $(7.24 \mathrm{ppm})$ in the $\mathrm{CDCl}_{3} \mathrm{NMR}$ solvent. Carbon chemical shifts are reported in parts per million and are calibrated to the solvent peaks $(77 \mathrm{ppm})$. Coupling constant values were extracted assuming first-order coupling. The multiplicities are abbreviated as follows: $\mathrm{s}=$ singlet, $\mathrm{d}=$ doublet, $\mathrm{t}=$ triplet, $\mathrm{q}=$ quartet, $\mathrm{m}=$ multiplet, and $\mathrm{br}=$ broad signal. High resolution mass spectra were obtained using an Agilent ESI-TOF mass spectrometer.

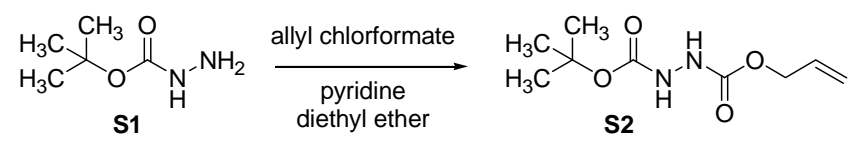

t-Butyl-allyloxycarbazate (S2): ${ }^{1}$ t-Butyl carbazate $\mathbf{S 1}(13.2 \mathrm{~g}, 100 \mathrm{mmol})$, pyridine $(8.9 \mathrm{~mL}, 110 \mathrm{mmol})$, and diethyl ether $\left(400 \mathrm{~mL}\right.$ ) were combined in a $1000 \mathrm{~mL}$ round-bottom flask under argon. The reaction mixture was then cooled to $0{ }^{\circ} \mathrm{C}$ and allylchloroformate $(10.61 \mathrm{~mL}, 100 \mathrm{mmol})$ was added dropwise over $5 \mathrm{~min}$. The mixture was then stirred at $0{ }^{\circ} \mathrm{C}$ for $45 \mathrm{~min}$ whereupon the reaction was warmed to ambient temperature and stirred for an additional $45 \mathrm{~min}$. The organics were then washed with $\mathrm{H}_{2} \mathrm{O}(1 \times 75 \mathrm{~mL}), 5 \% \mathrm{HCl}(1 \times 75 \mathrm{~mL})$, and $5 \% \mathrm{NaHCO}_{3}(1 \times 75 \mathrm{~mL})$. The combined organics were dried with $\mathrm{Na}_{2} \mathrm{SO}_{4}$ and the solvent was removed by rotary evaporation to provide $t$-butyl-allyloxycarbazate (S2) as a white solid (19.8 $\mathrm{g}$, 92\%). ${ }^{1} \mathrm{H}$ NMR (400 MHz, $\mathrm{CDCl}_{3}$ ) $\delta 6.55$ (s, 1H), 6.35 (s, 1H), 5.88 (m, 1H), 5.31 (d, 1H, J = 17.2 Hz), 5.21 (d, $1 \mathrm{H}, \mathrm{J}=10.4$ $\mathrm{Hz}$ ), 4.61 (dd, $2 \mathrm{H}, \mathrm{J}=1.2 \mathrm{~Hz}, \mathrm{~J}=5.6 \mathrm{~Hz}$ ); ${ }^{13} \mathrm{C} \mathrm{NMR}\left(100 \mathrm{MHz}, \mathrm{CDCl}_{3}\right) \delta$ 156.54, 155.66, 131.97, 118.29, 81.72, 66.49, 28.09; IR (thin film, $\mathrm{cm}^{-1}$ ) 3306, 2981, 1714, 1506, 1245, 1162. HRMS (EI) $\mathrm{C}_{9} \mathrm{H}_{16} \mathrm{~N}_{2} \mathrm{O}_{4}\left([\mathrm{M}]^{+}\right.$) 216.1110, found 216.1114.

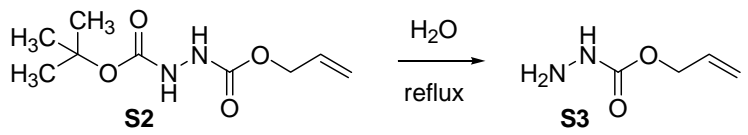

Allyloxycarbazate (S3): $t$-Butyl-allyloxyformyl carbazate S2 (30.85 g, $143 \mathrm{mmol})$ and $\mathrm{H}_{2} \mathrm{O}(200 \mathrm{~mL})$ were added to a 500 $\mathrm{mL}$ round-bottom flask equipped with a reflux condenser. The reaction flask was placed in an oil bath and heated to $160{ }^{\circ} \mathrm{C}$ for 2 hours. The mixture was allowed to cool to ambient temperature and then extracted with diethyl ether $(5 \times 50 \mathrm{~mL})$. The combined organics were dried with $\mathrm{Na}_{2} \mathrm{SO}_{4}$ and the solvent was removed by rotary evaporation to provide allyloxycarbazate (S3) as a clear oil (13.6 g, 82\%). ${ }^{1} \mathrm{H}$ NMR $\left(400 \mathrm{MHz}, \mathrm{CDCl}_{3}\right) \delta 6.61(\mathrm{~s}, 1 \mathrm{H}), 5.83(\mathrm{~m}, 1 \mathrm{H}), 5.22(\mathrm{~d}, 1 \mathrm{H}, \mathrm{J}=17.2 \mathrm{~Hz}), 5.14$ $(\mathrm{d}, 1 \mathrm{H}, \mathrm{J}=10.0 \mathrm{~Hz}), 4.52(\mathrm{~d}, 2 \mathrm{H}, \mathrm{J}=4.0 \mathrm{~Hz}), 3.77(\mathrm{~s}, 2 \mathrm{H}) ;{ }^{13} \mathrm{C}$ NMR $\left(100 \mathrm{MHz}, \mathrm{CDCl}_{3}\right) \delta$ 158.26, 132.14, 117.67, 65.72; IR (thin film, $\mathrm{cm}^{-1}$ ) 3340, 1716, 1647, 1506, 1277, 1182, 1064. HRMS (EI) $\mathrm{C}_{4} \mathrm{H}_{8} \mathrm{~N}_{2} \mathrm{O}_{2}$ ([M] $]^{+}$) 116.0586, found 116.0590.

\footnotetext{
${ }^{1}$ Gordon, M. S.; Krause, J. G.; Linneman-Mohr, M. A.; Parchue, R. R. Synthesis, 1980, 3, 244-245.
} 


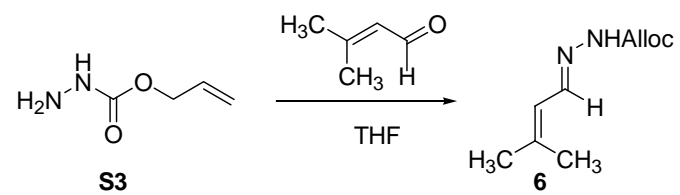

(3-Methyl-but-2-enylidene)-carbamic acid allyl ester (6): Allyloxycarbazate S3 (7.6, 65.1 mmol), 3-methyl-2-butenal $(6.9 \mathrm{~mL}, 71.6 \mathrm{mmol})$, and THF $(260 \mathrm{~mL})$ were added to a $1000 \mathrm{~mL}$ round-bottom flask and the reaction mixture was stirred for $16 \mathrm{~h}$. The solvent was removed by rotary evaporation and excess 3-methyl-2-butenal was removed in vacuo to provide 3methyl-but-2-enylidene)-carbamic acid allyl ester 6 as a clear oil $(11.1 \mathrm{~g}, 94 \%) .{ }^{1} \mathrm{H}$ NMR $\left(400 \mathrm{MHz}, \mathrm{CDCl}_{3}\right) \delta 7.87(\mathrm{~s}, 1 \mathrm{H})$, $7.72(\mathrm{~s}, 1 \mathrm{H}), 6.05$ (d, 1H, J = 9.2 Hz), $5.93(\mathrm{~m}, 1 \mathrm{H}), 5.32$ (d, 1H, J = $16.8 \mathrm{~Hz}$ ), 5.22 (dd, 1H, J = 1.2 Hz, J = 10.4 Hz), 4.69 $(\mathrm{m}, 2 \mathrm{H}), 1.85$ (d, 3H, J = 2.4 Hz), $1.82(\mathrm{~d}, 3 \mathrm{H}, \mathrm{J}=2.0 \mathrm{~Hz}) ;{ }^{13} \mathrm{C}$ NMR $\left(100 \mathrm{MHz}, \mathrm{CDCl}_{3}\right) \delta 153.66,144.47,132.03,121.63$, 117.57, 104.72, 65.53, 26.00, 18.25; IR (thin film, $\mathrm{cm}^{-1}$ ) 3224, 3049, 2973, 2912, 1721, 1648, 1543, 1376, 1248, 1032; HRMS (EI) $\mathrm{C}_{9} \mathrm{H}_{14} \mathrm{~N}_{2} \mathrm{O}_{2}\left([\mathrm{M}]^{+}\right)$182.1055, found 182.1054.

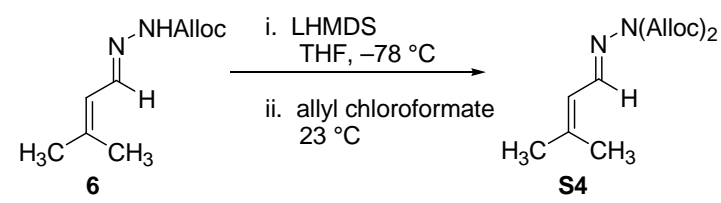

(3-Methyl-but-2-enylidene)-biscarbamic acid allyl ester (S4): (3-Methyl-but-2-enylidene)-carbamic acid allyl ester (6) $(11.1 \mathrm{~g}, 70 \mathrm{mmol})$ and THF $(350 \mathrm{~mL})$ were added to a flame dried $1 \mathrm{~L}$ round-bottom flask under argon. The mixture was cooled to $-78{ }^{\circ} \mathrm{C}$ and LHMDS in ( $1 \mathrm{M}$ solution in hexanes, $70 \mathrm{~mL}, 70 \mathrm{mmol}$ ) was added dropwise over $5 \mathrm{~min}$. The reaction was then stirred for an additional $30 \mathrm{~min}$ at $-78{ }^{\circ} \mathrm{C}$. Allylchloroformate $(7.85 \mathrm{~mL}, 74 \mathrm{mmol})$ was added in one portion and the reaction mixture was allowed to warm to ambient temperature and stirred for an additional $1.5 \mathrm{~h}$. The reaction mixture was concentrated by half by rotary evaportation and diethyl ether $(50 \mathrm{~mL})$ was added. The mixture was then washed with saturated $\mathrm{NaHCO}_{3}(2 \times 50 \mathrm{~mL})$. The organics were dried with $\mathrm{Na}_{2} \mathrm{SO}_{4}$ and the solvent was removed by rotary evaporation to provide a yellow oil. The residue was purified by flash chromatography (2:1 hexanes/ethyl acetate) to afford pure bisAlloc hydrazone S4 (12.2 g, 75\%). ${ }^{1} \mathrm{H}$ NMR $\left(400 \mathrm{MHz}, \mathrm{CDCl}_{3}\right) \delta 8.20(\mathrm{~d}, 1 \mathrm{H}, \mathrm{J}=10.0 \mathrm{~Hz}), 6.19(\mathrm{~d}, 1 \mathrm{H}, \mathrm{J}=10.0 \mathrm{~Hz}), 5.93(\mathrm{~m}$, $2 \mathrm{H}), 5.36(\mathrm{~d}, 2 \mathrm{H}, \mathrm{J}=16.8 \mathrm{~Hz}), 5.25(\mathrm{~d}, 2 \mathrm{H}, \mathrm{J}=10.4 \mathrm{~Hz}), 4.73(\mathrm{~d}, 4 \mathrm{H}, \mathrm{J}=5.6 \mathrm{~Hz}), 1.93(\mathrm{~s}, 3 \mathrm{H}), 1.89(\mathrm{~s}, 3 \mathrm{H}) ;{ }^{13} \mathrm{C}$ NMR $(100$ $\left.\mathrm{MHz}, \mathrm{CDCl}_{3}\right) \delta 164.85,152.12,151.59,131.29,121.44,118.99,67.82,26.85,19.13$; IR (thin film, $\mathrm{cm}^{-1}$ ) 1790, 1752, 1262, 1108; HRMS (EI) $\mathrm{C}_{13} \mathrm{H}_{18} \mathrm{~N}_{2} \mathrm{O}_{4}\left([\mathrm{M}]^{+}\right)$266.1267, found 266.1261 .

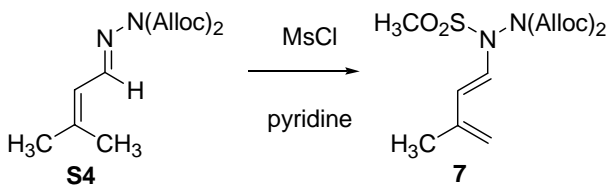

1-Hydrazinodiene (7): (3-Methyl-but-2-enylidene)-biscarbamic acid allyl ester $\mathbf{S 4}$ (1 g, $3.75 \mathrm{mmol}$ ) and pyridine (1.5 mL, $18.75 \mathrm{mmol}$ ) were added to a flame-dried $10 \mathrm{~mL}$ round-bottom flask. Methanesulfonic chloride $(0.87 \mathrm{~mL}, 11.25 \mathrm{mmol})$ was added dropwise over $1 \mathrm{~min}$ and the reaction was sealed and stirred at ambient temperature for $2 \mathrm{~h}$. The mixture was purified by flash chromatography (3:1 hexanes/ethyl acetate) to provide pure 1-hydrazinodiene $7(1.04 \mathrm{~g}, 80 \%) .{ }^{1} \mathrm{H} \mathrm{NMR}(400 \mathrm{MHz}$, $\left.\mathrm{CDCl}_{3}\right) \delta 6.63(\mathrm{~d}, 1 \mathrm{H}, \mathrm{J}=14.0 \mathrm{~Hz}), 5.91(\mathrm{~m}, 2 \mathrm{H}), 5.61(\mathrm{~d}, 1 \mathrm{H}, \mathrm{J}=14.0 \mathrm{~Hz}), 5.40(\mathrm{~d}, 2 \mathrm{H}, \mathrm{J}=16.4 \mathrm{~Hz}), 5.28(\mathrm{~d}, 2 \mathrm{H}, \mathrm{J}=9.8$ $\mathrm{Hz}$ ), 4.86 (d, 2H, J = $3.4 \mathrm{~Hz}$ ), $4.76(\mathrm{~m}, 4 \mathrm{H}), 3.16$ (s, 3H), 1.83 (s, 3H); ${ }^{13} \mathrm{C}$ NMR $\left(100 \mathrm{MHz}, \mathrm{CDCl}_{3}\right) \delta 151.14,138.79$, 130.46, 123.74, 119.5, 115.65, 114.00, 68.88, 41.50, 18.83; IR (thin film, $\mathrm{cm}^{-1}$ ) 1807, 1775, 1364, 1244, 1166, 1112; HRMS (ESI) $\mathrm{C}_{14} \mathrm{H}_{20} \mathrm{~N}_{2} \mathrm{O}_{6} \mathrm{SNa}\left([\mathrm{M}+\mathrm{Na}]^{+}\right)$367.0934, found 367.0926.

\section{Synthesis of Diels-Alder adduct 8}

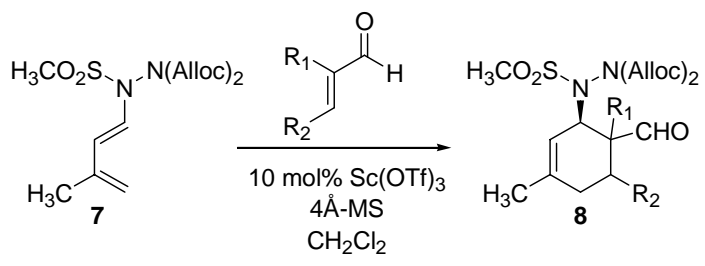


General procedure for the scandium(III) triflate $(\mathbf{1 0} \mathrm{mol} \%)$ catalyzed Diels-Alder reaction: Scandium(III) triflate (24.6 mg, $0.05 \mathrm{mmol})$ and $4 \AA$-molecular sieves $(75 \mathrm{mg}$ ) were added to a $15 \mathrm{~mL}$ recovery flask and the system was first flame dried under vacuum and then allowed to cool to ambient temperature under argon. 1-Hydrazinodiene 7 (172.5 mg, 0.5 mmol) and methylene chloride $(2.5 \mathrm{~mL})$ were added the flask and the mixture was stirred for $2 \mathrm{~min}$. The unsaturated aldehyde $(1.5 \mathrm{mmol})$ was then added and the reaction was sealed with a yellow Teflon cap. The reaction was stirred for the specified length of time at $23{ }^{\circ} \mathrm{C}$. The reaction mixture was then filtered through florisil, and the solvent was removed by rotary evaporation to provide a yellow oil. The residue was purified by flash chromatography to provide pure product 8 .

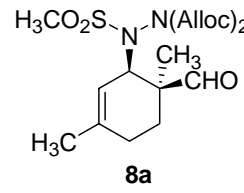

Methacrolein Diels-Alder adduct 8a: Reaction time: 3 hours. The product was isolated as a 5:1 mixture of inseparable isomers (185 mg, 89\%). The relative stereochemistry of the major diastereomer was determined by nOe studies of derivative 9a (see p S-5). ${ }^{1} \mathrm{H}$ NMR (500 MHz, $\left.\mathrm{CDCl}_{3}\right) \delta 9.96(\mathrm{~s}, 1 \mathrm{H})$, $5.89(\mathrm{~m}, 2 \mathrm{H}), 5.36-5.42(\mathrm{~m}, 3 \mathrm{H}), 5.24-5.30(\mathrm{~m}, 2 \mathrm{H}), 4.65-4.75(\mathrm{~m}, 3 \mathrm{H}), 4.53-4.57(\mathrm{~m}, 1 \mathrm{H}), 4.45$ (d, $1 \mathrm{H}, \mathrm{J}=4.5 \mathrm{~Hz}), 3.26(\mathrm{~s}, 3 \mathrm{H}), 1.94-2.07(\mathrm{~m}, 2 \mathrm{H}), 1.69-1.92(\mathrm{~m}, 2 \mathrm{H}), 1.66(\mathrm{~s}, 3 \mathrm{H}), 1.02(\mathrm{~s}, 3 \mathrm{H}) ;{ }^{13} \mathrm{C}$ NMR $\left(125 \mathrm{MHz}, \mathrm{CDCl}_{3}\right) \delta$ 204.96, 154.79, 152.74, 142.04, 130.81, 130.57, 119.81, 119.65, 115.20, 69.08, 68.48, 61.43, 47.11, 41.68, 26.14, 24.51, 23.42, 20.00; IR (thin film, $\mathrm{cm}^{-1}$ ) 2939, 1799, 1766, 1722, 1448, 1350, 1324, 1302, 1242, 1207, 1158, 1109, 992, 962, 937; HRMS (ESI) $\mathrm{C}_{18} \mathrm{H}_{26} \mathrm{~N}_{2} \mathrm{O}_{7} \mathrm{SNa}\left([\mathrm{M}+\mathrm{Na}]^{+}\right)$437.1353, found 437.1357.

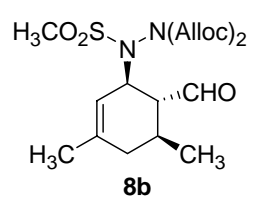

Crotonaldehyde Diels-Alder adduct 8b: Reaction time: 3 hours. The major isomer was isolated as a clear oil (135 mg, 65\%). The relative stereochemistry of the major diasteromer was determined by analogy with adduct 8d. ${ }^{1} \mathrm{H}$ NMR analysis of the crude reaction mixture reveals the aldehyde corresponding to the major exo-diasteromer is upfield from the endo-diasteromer. ${ }^{1} \mathrm{H}$ NMR $(400 \mathrm{MHz}$, $\left.\mathrm{CDCl}_{3}\right) \delta 9.85(\mathrm{~d}, 1 \mathrm{H}, \mathrm{J}=2 \mathrm{~Hz}), 5.88-5.98(\mathrm{~m}, 2 \mathrm{H}), 5.51(\mathrm{~s}, 1 \mathrm{H}), 5.39-5.44(\mathrm{~m}, 2 \mathrm{H}), 5.28-5.31(\mathrm{~m}$, 2H), $5.01(\mathrm{~d}, 1 \mathrm{H}, \mathrm{J}=10.0 \mathrm{~Hz}), 4.62-4.79(\mathrm{~m}, 4 \mathrm{H}), 3.08(\mathrm{~s}, 3 \mathrm{H}), 2.46-2.52(\mathrm{~m}, 1 \mathrm{H}), 1.80-1.91(\mathrm{~m}, 3 \mathrm{H}), 1.63(\mathrm{~s}, 3 \mathrm{H}), 1.04$ $(\mathrm{d}, 3 \mathrm{H}, \mathrm{J}=6.0 \mathrm{~Hz}) ;{ }^{13} \mathrm{C}$ NMR $\left(100 \mathrm{MHz}, \mathrm{CDCl}_{3}\right) \delta 204.21,153.47,153.26,139.07,130.60,119.84,119.57,68.97,68.82$, 58.17, 54.38, 41.81, 38.28, 32.67, 22.99, 18.98; IR (thin film, $\mathrm{cm}^{-1}$ ) 2963, 2934, 1802, 1771, 1742, 1446, 1352, 1326, 1241, 1158, 1110, 966; HRMS (ESI) $\mathrm{C}_{18} \mathrm{H}_{26} \mathrm{~N}_{2} \mathrm{O}_{7} \mathrm{SNa}\left([\mathrm{M}+\mathrm{Na}]^{+}\right)$437.1353, found 437.1345.

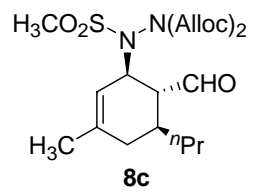

2-Hexenal Diels-Alder adduct 8c: Reaction time: 12 hours. The major isomer was isolated as a clear oil (100 mg, 45\%). The relative stereochemistry of the major diasteromer was determined by analogy with adduct 8d. ${ }^{1} \mathrm{H}$ NMR analysis of the crude reaction mixture reveals the aldehyde corresponding to the major exo-diasteromer is upfield from the endo-diasteromer. ${ }^{1} \mathrm{H} \mathrm{NMR}\left(500 \mathrm{MHz}, \mathrm{CDCl}_{3}\right) \delta 0.85$ (t, 3H, J $=7 \mathrm{~Hz}), 1.24-1.14(\mathrm{~m}, 1 \mathrm{H}), 1.32-1.24(\mathrm{~m}, 1 \mathrm{H}), 1.48-1.34(\mathrm{~m}, 2 \mathrm{H}), 1.63(\mathrm{~s}, 3 \mathrm{H}), 1.78-1.70(\mathrm{~m}, 1 \mathrm{H}), 1.90-$ $1.81(\mathrm{~m}, 1 \mathrm{H}), 1.98-1.92(\mathrm{~m}, 1 \mathrm{H}), 2.60-2.55(\mathrm{~m}, 1 \mathrm{H}), 3.06(\mathrm{~s}, 3 \mathrm{H}), 4.80-4.60(\mathrm{~m}, 4 \mathrm{H}), 5.10-4.95(\mathrm{~m}, 1 \mathrm{H}), 5.31-5.27(\mathrm{~m}, 2 \mathrm{H})$, 5.46-5.39 (m, 2H), $5.51(\mathrm{~s}, 1 \mathrm{H}), 5.98-5.88(\mathrm{~m}, 2 \mathrm{H}), 9.86$ (d, 1H, J = 2.5 Hz); ${ }^{13} \mathrm{C}$ NMR $\left(125 \mathrm{MHz}, \mathrm{CDCl}_{3}\right) \delta 14.05,19.31$, 23.15, 34.77, 35.11, 37.59, 41.81, 52.95, 58.46, 68.85, 69.00, 119.55, 119.61, 119.87, 130.63, 138.92, 153.27, 153.53, 184.54, 189.65, 204.61; IR (thin film, $\mathrm{cm}^{-1}$ ) 2959, 2933, 1801, 1770, 1352, 1240, 1158, 1100; HRMS (ESI) $\mathrm{C}_{20} \mathrm{H}_{30} \mathrm{~N}_{2} \mathrm{O}_{7} \mathrm{SNa}$ $(\mathrm{M}+\mathrm{Na})^{+} 465.1666$, found $465.1656 \mathrm{~m} / \mathrm{z}$.

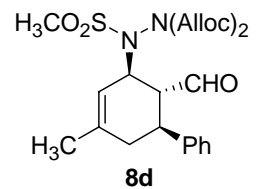

Cinnamaldehyde Diels-Alder adduct 8d: Reaction time: 3 hours. The major isomer was isolated as a white solid (173 mg, 72\%). The relative stereochemistry of the major diastereomer was determined by $\mathrm{x}$ ray crystallographic analysis. See p S-15 for crystal structure data. ${ }^{1} \mathrm{H}$ NMR $\left(500 \mathrm{MHz}, \mathrm{CDCl}_{3}\right) \delta 9.30$ (s, $1 \mathrm{H}), 7.31$ (t, 2H, J = 7.5 Hz), $7.19-7.25$ (m, 3H), 5.94 (m, 2H), 5.65 (s, 1H), 5.39 - 5.48 (m, 2H), 5.28 $5.32(\mathrm{~m}, 2 \mathrm{H}), 5.16(\mathrm{~d}, 1 \mathrm{H}, \mathrm{J}=8.0 \mathrm{~Hz}), 4.66-4.79(\mathrm{~m}, 4 \mathrm{H}), 3.17(\mathrm{dt}, 1 \mathrm{H}, \mathrm{J}=1.5 \mathrm{~Hz}, \mathrm{~J}=6.0 \mathrm{~Hz}), 3.06$ (s, 3H), $2.87(\mathrm{dt}, 1 \mathrm{H}, \mathrm{J}=4.5 \mathrm{~Hz}, \mathrm{~J}=12.0 \mathrm{~Hz}), 2.33(\mathrm{t}, 1 \mathrm{H}, \mathrm{J}=15.0 \mathrm{~Hz}), 2.04(\mathrm{dd}, 1 \mathrm{H}, \mathrm{J}=4.5 \mathrm{~Hz}, \mathrm{~J}=17.5 \mathrm{~Hz}), 1.68(\mathrm{~s}, 3 \mathrm{H}) ;{ }^{13} \mathrm{C}$ NMR (125 MHz, CDCl $) \delta$ 203.34, 153.70, 153.01, 140.32, 138.74, 130.65, 130.57, 124.02, 127.49, 120.24, 120.16, 119.69, 69.10, 68.90, 58.10, 52.68, 44.81, 41.99; IR (thin film, $\mathrm{cm}^{-1}$ ) 2914, 1801, 1769, 1739, 1453, 1352, 1327, 1240, 1156, 1109, 1032, 966; HRMS (ESI) $\mathrm{C}_{23} \mathrm{H}_{28} \mathrm{~N}_{2} \mathrm{O}_{7} \mathrm{SNa}(\mathrm{M}+\mathrm{Na})^{+}$499.1509, found $499.1497 \mathrm{~m} / \mathrm{z}$.

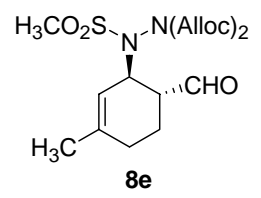

Acrolein Diels-Alder adduct 8e: Reaction time: 3 hours. The major isomer was isolated as a clear oil (94 mg, 47\%). The relative stereochemistry of the major diasteromer was determined by analogy with adduct 8d. ${ }^{1} \mathrm{H}$ NMR analysis of the crude reaction mixture reveals the aldehyde corresponding to the major exo-diasteromer is upfield from the endo-diasteromer. ${ }^{1} \mathrm{H}$ NMR $\left(500 \mathrm{MHz}, \mathrm{CDCl}_{3}\right) \delta 9.67(\mathrm{~s}, 1 \mathrm{H})$, 5.86 - 5.94 (m, 2H), 5.45 (s, 1H), 5.38 - $5.43(\mathrm{~m}, 2 \mathrm{H}), 5.28(\mathrm{~d}, 2 \mathrm{H}, \mathrm{J}=10.0 \mathrm{~Hz}), 4.95(\mathrm{~s}, 1 \mathrm{H}), 4.64-4.72$ (m, 4H), 3.16 (s, 3H), $2.81-2.85(\mathrm{~m}, 1 \mathrm{H}), 1.93-2.01(\mathrm{~m}, 2 \mathrm{H}), 1.83-1.87(\mathrm{~m}, 1 \mathrm{H}), 1.66-1.70(\mathrm{~m}, 1 \mathrm{H}), 1.64(\mathrm{~s}, 3 \mathrm{H}) ;{ }^{13} \mathrm{C}$ 
NMR (125 MHz, $\left.\mathrm{CDCl}_{3}\right) \delta 201.65,153.48,153.34,141.25,130.59,130.56,119.92,119.68,118.20,68.93,68.87,56.01$, 48.63, 41.91, 27.24, 23.47, 21.59; IR (thin film, $\mathrm{cm}^{-1}$ ) 3021, 2936, 1802, 1769, 1740, 1449, 1351, 1328, 1242, 1215, 1155, 1108; HRMS (ESI) $\mathrm{C}_{17} \mathrm{H}_{24} \mathrm{~N}_{2} \mathrm{O}_{7} \mathrm{SNa}(\mathrm{M}+\mathrm{Na})^{+} 423.1196$, found $423.1177 \mathrm{~m} / \mathrm{z}$.

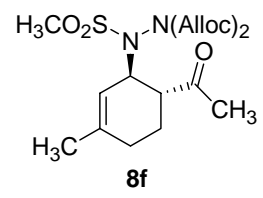

Methyl vinyl ketone Diels-Alder adduct 8f: Reaction time: 3 hours. The major isomer was isolated as a clear oil (136 mg, 66\%). The relative stereochemistry of the major diasteromer was determined by coupling constants of derivative $9 f$ (see p S-6). ${ }^{1} \mathrm{H}$ NMR $\left(500 \mathrm{MHz}, \mathrm{CDCl}_{3}\right) \delta 5.87-$ $5.93(\mathrm{~m}, 2 \mathrm{H}), 5.49(\mathrm{~s}, 1 \mathrm{H}), 5.37-5.42(\mathrm{~m}, 2 \mathrm{H}), 5.26-5.27(\mathrm{~m}, 2 \mathrm{H}), 4.90(\mathrm{~d}, 1 \mathrm{H}, \mathrm{J}=8 \mathrm{~Hz}), 4.62-$ $4.74(\mathrm{~m}, 3 \mathrm{H}), 4.58-4.60(\mathrm{~m}, 1 \mathrm{H}), 3.08(\mathrm{~s}, 3 \mathrm{H}), 2.79-2.84(\mathrm{~m}, 1 \mathrm{H}), 2.14(\mathrm{~s}, 3 \mathrm{H}), 1.92-2.04(\mathrm{~m}$, 2H), 1.80 - 1.84 (m, 1H), 1.61 (s, 3H), $1.46-1.54(\mathrm{~m}, 1 \mathrm{H}) ;{ }^{13} \mathrm{C}$ NMR (125 MHz, CDCl $) \delta 208.63,153.80,153.01,139.30$, 130.61, 119.88, 119.52, 119.41, 68.87, 68.68, 57.21, 49.50, 41.73, 28.53, 28.28, 25.50, 23.21; IR (thin film, $\mathrm{cm}^{-1}$ ) 3020, 2936, 1800, 1769, 1743, 1713, 1650, 1449, 1350, 1327, 1303, 1244, 1155, 1113, 1034, 965; HRMS (ESI) $\mathrm{C}_{18} \mathrm{H}_{26} \mathrm{~N}_{2} \mathrm{O}_{7} \mathrm{SNa}$ $(\mathrm{M}+\mathrm{Na})^{+}$437.1353, found $437.1342 \mathrm{~m} / \mathrm{z}$.

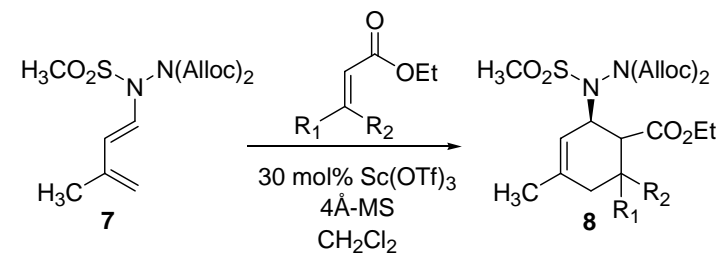

General procedure for the scandium(III) triflate (30 mol\%) catalyzed Diels-Alder reaction: Scandium(III) triflate (185 $\mathrm{mg}, 0.43 \mathrm{mmol})$ and $4 \AA$-molecular sieves $(215 \mathrm{mg})$ were added to a $15 \mathrm{~mL}$ recovery flask and the system was flame dried under vacuum and then allowed to cool to ambient temperature under argon. 1-Hydrazinodiene 7 (500 mg, $1.4 \mathrm{mmol}$ ) and methylene chloride $(4 \mathrm{~mL})$ were added the flask and the mixture was stirred for $2 \mathrm{~min}$. The unsaturated ester (4.3 mmol) was then added and the reaction was sealed with a yellow Teflon cap. The reaction was stirred for the specified length of time at $23^{\circ} \mathrm{C}$. The reaction mixture was then filtered through florisil, and the solvent was removed by rotary evaporation to provide a yellow oil. The residue was purified by flash chromatography to provide pure product $\mathbf{8}$.

$\mathrm{H}_{3} \mathrm{CO}_{2} \mathrm{~S}_{\mathrm{N}^{-}} \mathrm{N}(\mathrm{Alloc})_{2}$ Diethyl maleate Diels-Alder adduct 8g: Reaction time: 15 hours. The product was purified by flash . $\mathrm{CO}_{2} \mathrm{Et}$ chromatography (3:1 hexanes/ethyl acetate) to provide $\mathbf{8 g}$ as a clear oil $(567 \mathrm{mg}$, $76 \%$ ). The relative stereochemistry of the major diasteromer was determined by nOe studies of 13a. ${ }^{1} \mathrm{H}$ NMR $(500 \mathrm{MHz}$, $\left.\mathrm{CDCl}_{3}\right) \delta 1.18(\mathrm{t}, 3 \mathrm{H}, \mathrm{J}=7 \mathrm{~Hz}), 1.23(\mathrm{t}, 3 \mathrm{H}, \mathrm{J}=7 \mathrm{~Hz}), 1.68(\mathrm{~s}, 3 \mathrm{H}), 2.19(\mathrm{dd}, 1 \mathrm{H}, \mathrm{J}=6,18.5 \mathrm{~Hz}), 2.33$ (dd, $1 \mathrm{H}, \mathrm{J}=10,18.5 \mathrm{~Hz}$ ), 3.08 (ddd, $1 \mathrm{H}, \mathrm{J}=4,6,10 \mathrm{~Hz}$ ), 3.19 (s, 3H), 3.70-3.67 (m, 1H), 4.10-4.05 (m, 2H), 4.16-4.11 (m, 2H), 4.77-4.64 (m, 4H), $4.98(\mathrm{~s}, 1 \mathrm{H}), 5.29-5.24(\mathrm{~m}, 2 \mathrm{H}), 5.35-5.33,1 \mathrm{H}), 5.43-5.36(\mathrm{~m}, 2 \mathrm{H}), 5.96-5.85(\mathrm{~m}$, 2H); ${ }^{13} \mathrm{C}$ NMR (125 MHz, $\left.\mathrm{CDCl}_{3}\right) \delta 13.93,14.12,23.56,28.83,37.16,42.68,44.36,57.67,60.70,61.07,68.83,68.86$, 115.71, 119.63, 119.71, 130.60, 141.95, 153.06, 170.37, 172.76; IR (thin film, $\mathrm{cm}^{-1}$ ) 2983, 2939, 1804, 1770, 1732, 1447, 1354, 1242, 1108, 1033; HRMS (ESI) $\mathrm{C}_{22} \mathrm{H}_{32} \mathrm{~N}_{2} \mathrm{O}_{10} \mathrm{SNa}(\mathrm{M}+\mathrm{Na})^{+} 539.167$, found $539.1674 \mathrm{~m} / \mathrm{z}$.

$\mathrm{H}_{3} \mathrm{CO}_{2} \mathrm{~S}_{\mathrm{N}^{-}} \mathrm{N}(\mathrm{Allloc})_{2}$ Diethyl fumarate Diels-Alder adduct 8h: Reaction time: 16 hours. The product was purified by flash chromatography (3:1 hexanes/ethyl acetate) to provide $\mathbf{8 h}$ as a clear oil (225 $\mathrm{mg}, 30 \%)$. The relative stereochemistry of the major diasteromer was determined by nOe studies of 13b. ${ }^{1} \mathrm{H}$ NMR $(500 \mathrm{MHz}$, $\left.\mathrm{CDCl}_{3}\right) \delta 1.21(\mathrm{t}, 6 \mathrm{H}, \mathrm{J}=7 \mathrm{~Hz}), 1.63(\mathrm{~s}, 3 \mathrm{H}), 2.02(\mathrm{dd}, 1 \mathrm{H}, \mathrm{J}=4.5,17 \mathrm{~Hz}), 2.36-2.38(\mathrm{~m}, 1 \mathrm{H}), 2.84-2.73$ (m, 2H), 313 (s, 3H), 4.14-3.99 (m, 4H), 4.76-4.59 (m, 4H), 5.03-4.99 (m, 1H), 5.26 (d, 2H, J = 9.5 Hz), 5.38-5.35 (m, 1H), 5.42-5.39 (m, 1H), 5.59 (s, broad, 1H), 5.95-5.85 (m, 2H); ${ }^{13} \mathrm{C}$ NMR (125 MHz, CDCl 3 ) $\delta 13.88,13.98$, 22.86, 31.62, 41.75, 44.39, 44.86, 60.58, 60.92, 61.24, 68.75, 68.86, 119.41, 119.48, 120.21, 130.69, 136.69, 153.18, 153.48, 172.26, 172.61; IR (thin film, $\mathrm{cm}^{-1}$ ) 2984, 2939, 1802, 1770, 1738, 1446, 1353, 1240, 1157, 1113, 1033; HRMS (ESI) $\mathrm{C}_{22} \mathrm{H}_{32} \mathrm{~N}_{2} \mathrm{O}_{10} \mathrm{SNa}(\mathrm{M}+\mathrm{Na})^{+} 539.1670$, found $539.1676 \mathrm{~m} / \mathrm{z}$. 


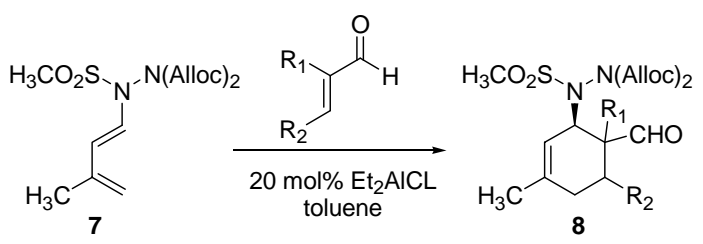

General procedure for the diethylaluminum chloride catalyzed Diels-Alder reaction: 1 -Hydrazinodiene 7 (172.5 mg, $0.5 \mathrm{mmol}$ ) and toluene $(2.5 \mathrm{~mL})$ were added to a flame dried, $15 \mathrm{~mL}$ recovery flask under argon. The unsaturated aldehyde was added followed by diethyl aluminum chloride (1.8 M solution in toluene, $56 \mu \mathrm{L}, 0.1 \mathrm{mmol})$. The reaction was sealed with a yellow Teflon cap and stirred for the specified length of time at $23^{\circ} \mathrm{C}$. The reaction mixture was then filtered through florisil, and the solvent was removed by rotary evaporation to provide a yellow oil. The residue was purified by flash chromatography to provide pure product 8 .

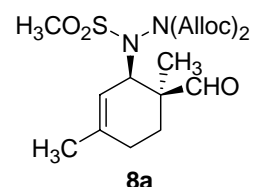

$8 \mathrm{a}$

Methacrolein Diels-Alder adduct 8a: Reaction time: 2.5 hours. The product was isolated as a 12:1 mixture of inseparable isomers (169 mg, 81\%). The relative stereochemistry of the major diastereomer was determined by nOe studies of derivative 9a (see p S-5). ${ }^{1} \mathrm{H}$ NMR $\left(500 \mathrm{MHz}, \mathrm{CDCl}_{3}\right) \delta 9.96(\mathrm{~s}, 1 \mathrm{H})$, $5.89(\mathrm{~m}, 2 \mathrm{H}), 5.36-5.42(\mathrm{~m}, 3 \mathrm{H}), 5.24-5.30(\mathrm{~m}, 2 \mathrm{H}), 4.65-4.75(\mathrm{~m}, 3 \mathrm{H}), 4.53-4.57(\mathrm{~m}, 1 \mathrm{H}), 4.45$ (d, $1 \mathrm{H}, \mathrm{J}=4.5 \mathrm{~Hz}), 3.26$ (s, 3H), $1.94-2.07$ (m, 2H), $1.69-1.92(\mathrm{~m}, 2 \mathrm{H}), 1.66(\mathrm{~s}, 3 \mathrm{H}), 1.02(\mathrm{~s}, 3 \mathrm{H}) ;{ }^{13} \mathrm{C}$ NMR $\left(125 \mathrm{MHz}, \mathrm{CDCl}_{3}\right) \delta$ 204.96, 154.79, 152.74, 142.04, 130.81, 130.57, 119.81, 119.65, 115.20, 69.08, 68.48, 61.43, 47.11, 41.68, 26.14, 24.51, 23.42, 20.00; IR (thin film, $\mathrm{cm}^{-1}$ ) 2939, 1799, 1766, 1722, 1448, 1350, 1324, 1302, 1242, 1207, 1158, 1109, 992, 962, 937; HRMS (ESI) $\mathrm{C}_{18} \mathrm{H}_{26} \mathrm{~N}_{2} \mathrm{O}_{7} \mathrm{~S}\left([\mathrm{M}+\mathrm{Na}]^{+}\right)$437.1353, found 437.1357.

\section{Deprotection of Diels-Alder adduct 8}

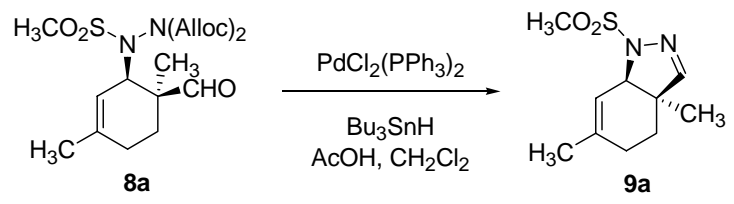

1-Methanesulfonyl-3 $\alpha, 6$-dimethyl-3 $\boldsymbol{\alpha}, 4,5,7 \boldsymbol{\alpha}$-tetrahydro-1H-indazole (9a): Diels-Alder adduct 8a (150 mg, $0.36 \mathrm{mmol}$ ), acetic acid (98.9 $\mu \mathrm{L}, 1.7 \mathrm{mmol})$, bistriphenylphosphinepalladium dichloride (10.1 mg, $0.014 \mathrm{mmol})$, and methylene chloride ( $7.2 \mathrm{~mL}$ ) were added to a $25 \mathrm{~mL}$ round-bottom flask under argon. Tributyltin hydride (212.5 $\mu \mathrm{L}, 0.79$ mmol) was added in one portion and the reaction was stirred for $1 \mathrm{~h}$. The product was purified by flash chromatography (first hexanes, then 1:1 hexanes/ethyl acetate) to provide a clear oil. The product was purified two more times by flash chromatography (1:1 hexanes/ethyl acetate) to provide 1-methanesulfonyl-3 $\alpha, 6$-dimethyl-3 $\alpha, 4,5,7 \alpha$-tetrahydro-1H-indazole as a clear oil (56 mg, 68\%). ${ }^{1} \mathrm{H}$ NMR (500 MHz, $\left.\mathrm{CDCl}_{3}\right) \delta 6.92(\mathrm{~s}, 1 \mathrm{H}), 5.72(\mathrm{~s}, 1 \mathrm{H}), 3.91$ (m, 1H), $3.06(\mathrm{~s}, 3 \mathrm{H}), 1.98-2.03$ (m, 2H), $1.78(\mathrm{~s}, 3 \mathrm{H})$, $1.68-1.76(\mathrm{~m}, 1 \mathrm{H}), 1.60-1.66(\mathrm{~m}, 1 \mathrm{H}), 1.23(\mathrm{~s}, 3 \mathrm{H}) ;{ }^{13} \mathrm{C} \mathrm{NMR}\left(125 \mathrm{MHz}, \mathrm{CDCl}_{3}\right) \delta 157.50,139.92,116.88,64.50,48.49$, 37.21, 28.22, 26.14, 23.74, 20.94; IR (thin film, $\mathrm{cm}^{-1}$ ) 2967, 2932, 1672, 1602, 1447, 1348, 1163, 1071, 982; HRMS (ESI) $\mathrm{C}_{10} \mathrm{H}_{16} \mathrm{~N}_{2} \mathrm{O}_{2} \mathrm{~S}\left([\mathrm{M}+\mathrm{Na}]^{+}\right)$251.0830, found 251.0811.

\section{The following selected nOe was observed for heterocycle 9a:}

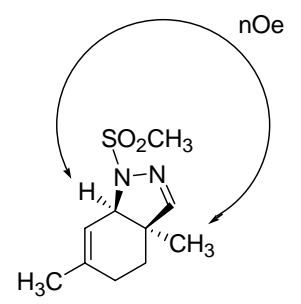



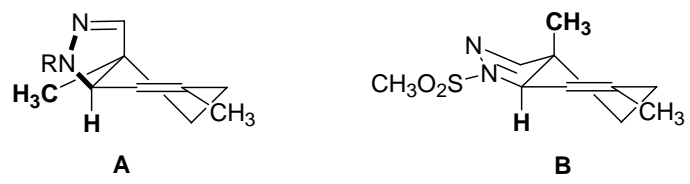

nOe observed

This nOe can only be observed with a cis-fused ring system (A). If the rings were trans-fused (B), the methyl group and the methyne proton would both be pseudoaxial and no nOe would be observed.

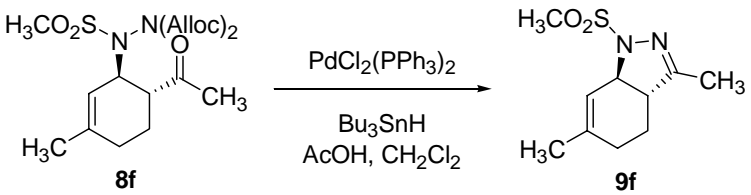

1-Methanesulfonyl-3,6-dimethyl-3 $\alpha, 4,5,7 \boldsymbol{\alpha}$-tetrahydro-1H-indazole (9f): Diels-Alder adduct 8 (33 mg, $0.08 \mathrm{mmol}$ ), acetic acid (22.0 $\mu \mathrm{L}, 0.38 \mathrm{mmol})$, bistriphenylphosphinepalladium dichloride ( $2.3 \mathrm{mg}, 0.0032 \mathrm{mmol})$, and methylene chloride $(1.6 \mathrm{~mL})$ were added to a $25 \mathrm{~mL}$ round-bottom flask under argon. Tributyltin hydride $(47.2 \mu \mathrm{L}, 0.18 \mathrm{mmol})$ was added in one portion and the reaction was stirred for $1 \mathrm{~h}$. The product was purified by flash chromatography (first hexanes, then 1:1 hexanes/ethyl acetate) to provide a white solid. The product was purified again by flash chromatography (1:1 hexanes/ethyl

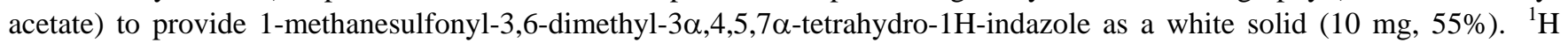
NMR (500 MHz, CDCl $) \delta 1.59-1.62(\mathrm{~m}, 1 \mathrm{H}), 1.67$ (s, 3H), 2.04 (s, 3H), 2.11-2.19 (m, 3H), 2.65 (t, 1H, J = 14 Hz), 2.97 (s, 3H), $3.67(\mathrm{~d}, 1 \mathrm{H}, \mathrm{J}=14 \mathrm{~Hz}), 6.09(\mathrm{~s}, 1 \mathrm{H}) ;{ }^{13} \mathrm{C} \mathrm{NMR}\left(125 \mathrm{MHz}, \mathrm{CDCl}_{3}\right) \delta 14.44,21.89,22.70,31.17,34.81,51.87,66.34$, 120.72, 137.71, 163.31; IR (thin film, $\mathrm{cm}^{-1}$ ) 853.9, 961.9, 1054.8, 1076.9, 1163.2, 1342.7, 1433.0, 2916.3.
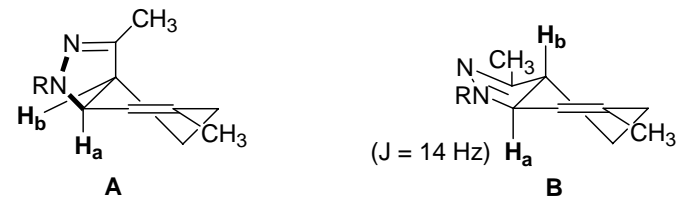

If the rings were cis-fused (A), there would be a small coupling constant between $\mathrm{H}_{\mathrm{a}}$ and $\mathrm{H}_{\mathrm{b}}$. However, the large observed coupling constant $\left(\mathrm{J}=14 \mathrm{~Hz}\right.$ ) is consistent with the vicinal, axial relationship of $\mathrm{H}_{\mathrm{a}}$ and $\mathrm{H}_{\mathrm{b}}$ in the trans-fused rings (B).

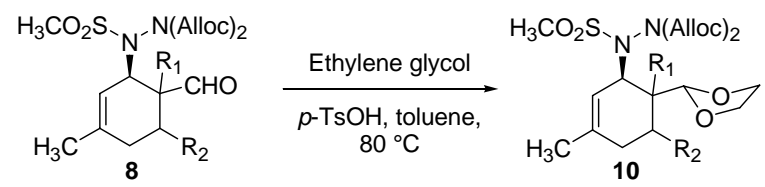

General procedure for the acetal protection of Diels-Alder adduct 8: Diels-Alder adduct 8, $p$-toluenesulfonic acid (5 mol\%), and 1:5 toluene/ethylene glycol $(0.25 \mathrm{M})$ were added to a $10 \mathrm{~mL}$ round-bottom flask equipped with a reflux condenser. The reaction was then heated to $80^{\circ} \mathrm{C}$ for $2 \mathrm{~h}$. The reaction mixture was then poured into saturated $\mathrm{NaHCO}_{3}(10$ $\mathrm{mL})$ and extracted with methylene chloride $(3 \times 20 \mathrm{~mL})$. The combined organics were dried with $\mathrm{Na}_{2} \mathrm{SO}_{4}$ and the solvent was removed by rotary evaporation to provide a clear oil. The residue was purified by flash chromatography (3:1 hexanes/ethyl acetate) to provide pure acetal $\mathbf{1 0 .}$

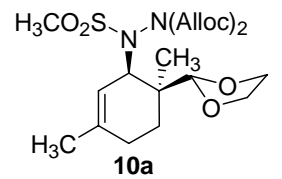

Acetal 10a: The reaction was run with $0.34 \mathrm{mmol}$ 8a. The product was isolated as a clear oil $(146 \mathrm{mg}$, 79\%). ${ }^{1} \mathrm{H}$ NMR (500 MHz, $\left.\mathrm{CDCl}_{3}\right) \delta 5.84-5.94(\mathrm{~m}, 2 \mathrm{H}), 5.35-5.43(\mathrm{~m}, 3 \mathrm{H}), 5.24-5.28(\mathrm{~m}, 3 \mathrm{H})$, $4.61-4.73(\mathrm{~m}, 4 \mathrm{H}), 4.21(\mathrm{~d}, 1 \mathrm{H}, \mathrm{J}=6 \mathrm{~Hz}), 3.87-3.94(\mathrm{~m}, 2 \mathrm{H}), 3.78-3.85(\mathrm{~m}, 2 \mathrm{H}), 3.25(\mathrm{~s}, 3 \mathrm{H})$, $1.89-2.03(\mathrm{~m}, 2 \mathrm{H}), 1.65$ (s, 3H), $1.49-1.53(\mathrm{~m}, 1 \mathrm{H}), 0.82(\mathrm{~s}, 3 \mathrm{H}) ;{ }^{13} \mathrm{C}$ NMR $\left(125 \mathrm{MHz}, \mathrm{CDCl}_{3}\right) \delta$ 153.88, 153.54, 139.96, 130.86, 130.68, 119.29, 119.14, 117.53, 105.75, 68.71, 68.53, 65.67, 64.85, 
61.50, 42.12, 40.16, 26.00, 24.30; IR (thin film, $\mathrm{cm}^{-1}$ ) 2945, 2886, 1764, 1745, 1448, 1346, 1319, 1240, 1193, 1145, 1100, 977, 932; HRMS (ESI) $\mathrm{C}_{20} \mathrm{H}_{30} \mathrm{~N}_{2} \mathrm{O}_{8}$ Sna $\left([\mathrm{M}+\mathrm{Na}]^{+}\right)$481.1615, found 481.1623.

$\mathrm{H}_{3} \mathrm{CO}_{2} \mathrm{~S}_{\mathrm{N}^{-}} \mathrm{N}(\mathrm{Allloc})_{2} \quad$ Acetal 10b: The reaction was run with $0.27 \mathrm{mmol} \mathbf{8 b}$. The product was isolated as a clear oil (90.8 $\mathrm{mg}$, 75\%). ${ }^{1} \mathrm{H}$ NMR (500 MHz, $\left.\mathrm{CDCl}_{3}\right) \delta 5.21(\mathrm{~s}, 1 \mathrm{H}), 4.92(\mathrm{~d}, 1 \mathrm{H}, \mathrm{J}=3.0 \mathrm{~Hz}), 4.70(\mathrm{~d}, 1 \mathrm{H}, \mathrm{J}=14 \mathrm{~Hz})$, 3.82 - 3.99 (m, 4H), 3.32 (s, 2H), 3.00 (s, 3H), 1.88 - 2.02 (m, 4H), 1.71 (s, 3H), 1.52 - $1.58(\mathrm{~m}, 1 \mathrm{H})$; ${ }^{13} \mathrm{C}$ NMR $\left(125 \mathrm{MHz}, \mathrm{CDCl}_{3}\right) \delta 143.33,119.65,104.32$, 65.38, 65.02, 56.69, 39.01, 37.84, 29.35, 23.39, 21.42; IR (thin film, $\mathrm{cm}^{-1}$ ) 3368, 2928, 2896, 1307, 1152, 1035, 957. HRMS (ESI) $\mathrm{C}_{20} \mathrm{H}_{30} \mathrm{~N}_{2} \mathrm{O}_{8} \mathrm{Sna}(\mathrm{M}+\mathrm{Na})^{+} 481.1615$, found $481.1607 \mathrm{~m} / \mathrm{z}$.

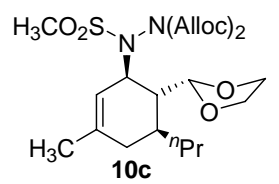

Acetal 10c: The reaction was run with $0.3 \mathrm{mmol} 8 \mathrm{c}$. The product was isolated as a clear oil $(145 \mathrm{mg}$, 98\%). ${ }^{1} \mathrm{H}$ NMR $\left(300 \mathrm{MHz}, \mathrm{CDCl}_{3}\right) \delta 0.82(\mathrm{t}, 3 \mathrm{H}, \mathrm{J}=7 \mathrm{~Hz}), 1.24-1.08(\mathrm{~m}, 2 \mathrm{H}), 1.44-1.28(\mathrm{~m}, 2 \mathrm{H}), 1.63$ (s, 3H), 1.82-1.66 (m, 2H), 1.98-1.88 (m, 1H), 2.16-2.08 (m, 1H), $3.22(\mathrm{~s}, 3 \mathrm{H}), 3.85-3.75(\mathrm{~m}, 2 \mathrm{H})$, 4.00-3.90 (m, 2H), 4.80-4.54 (m, 5H), 5.01 (d, 1H, J = 2 Hz), 5.28-5.21 (m, 2H), 5.37 (s, 1H), $5.44-$ $5.40(\mathrm{~m}, 2 \mathrm{H}), 5.98-5.82(\mathrm{~m}, 2 \mathrm{H}) ;{ }^{13} \mathrm{C}$ NMR $\left(75 \mathrm{MHz}, \mathrm{CDCl}_{3}\right) \delta 14.22,19.85,23.82,32.57,33.60$, 36.24, 41.81, 42.86, 58.02, 64.46, 64.67, 68.58, 68.64, 104.82, 118.37, 119.23, 130.67, 130.83, 140.97, 153.45, 153.73; IR (thin film, $\mathrm{cm}^{-1}$ ) 2958, 2932, 1800, 1767, 1349, 1240, 1157; HRMS (ESI) $\mathrm{C}_{22} \mathrm{H}_{34} \mathrm{~N}_{2} \mathrm{O}_{8} \mathrm{Sna}(\mathrm{M}+\mathrm{Na})^{+} 509.1928$, found $509.1930 \mathrm{~m} / \mathrm{z}$.

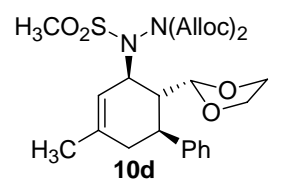

Acetal 10d: The reaction was run with $0.29 \mathrm{mmol} \mathbf{8 d}$. The product was isolated as a clear oil $(127 \mathrm{mg}$, 88\%). ${ }^{1} \mathrm{H}$ NMR $\left(500 \mathrm{MHz}, \mathrm{CDCl}_{3}\right) \delta 7.26-7.28(\mathrm{~m}, 2 \mathrm{H}), 7.17-7.22(\mathrm{~m}, 3 \mathrm{H}), 5.98(\mathrm{~m}, 2 \mathrm{H}), 5.27(\mathrm{~s}$, $1 \mathrm{H}), 5.43-5.50(\mathrm{~m}, 2 \mathrm{H}), 5.29-5.34(\mathrm{~m}, 2 \mathrm{H}), 5.05(\mathrm{~s}, 1 \mathrm{H}), 4,71-4.85(\mathrm{~m}, 4 \mathrm{H}), 3.85(\mathrm{~m}, 1 \mathrm{H}), 3.73(\mathrm{~m}$, $1 \mathrm{H}), 3.57(\mathrm{~m}, 1 \mathrm{H}), 3.28(\mathrm{~s}, 3 \mathrm{H}), 3.17(\mathrm{~m}, 1 \mathrm{H}), 2.99(\mathrm{~m}, 1 \mathrm{H}), 2.70(\mathrm{~m}, 1 \mathrm{H}), 2.29(\mathrm{t}, 1 \mathrm{H}, \mathrm{J}=13.0 \mathrm{~Hz}), 1.98$ $(\mathrm{m}, 1 \mathrm{H}), 1.74(\mathrm{~s}, 3 \mathrm{H}) ;{ }^{13} \mathrm{C}$ NMR $\left(125 \mathrm{MHz}, \mathrm{CDCl}_{3}\right) \delta 153.98,153.54,144.31,142.30,130.95,130.74$, 128.04, 127.98, 126.07, 119.42, 119.01, 103.55, 68.83, 68.76, 65.04, 64.14, 59.64, 43.75, 42.48, 42.16, 37.86, 23.37; IR (thin film, $\mathrm{cm}^{-1}$ ) 3028, 2891, 1798, 1766, 1352, 1323, 1301, 1240, 1205, 1157, 1116, 1030, 961; HRMS (ESI) $\mathrm{C}_{25} \mathrm{H}_{32} \mathrm{~N}_{2} \mathrm{O}_{8}$ Sna $(\mathrm{M}+\mathrm{Na})^{+}$543.1771, found $543.1768 \mathrm{~m} / \mathrm{z}$.

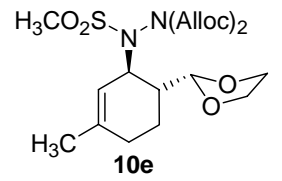

Acetal 10e: The reaction was run with $0.27 \mathrm{mmol} \mathbf{8 e}$. The product was isolated as a clear oil (109.1 mg, 93\%). ${ }^{1} \mathrm{H}$ NMR (500 MHz, $\left.\mathrm{CDCl}_{3}\right) \delta 5.86-5.96(\mathrm{~m}, 2 \mathrm{H}), 5.36-5.44(\mathrm{~m}, 2 \mathrm{H}), 5.35(\mathrm{~s}, 1 \mathrm{H}), 5.25$ $5.28(\mathrm{~m}, 1 \mathrm{H}), 5.07$ (d, 2H, J = 4.0 Hz), $4.64-4.77$ (m, 4H), 4.58 (m, 1H), $3.81-3.97$ (m, 4H), 3.20 (s, $3 \mathrm{H}), 2.12-2.17(\mathrm{~m}, 1 \mathrm{H}), 1.86-1.90(\mathrm{~m}, 2 \mathrm{H}), 1.70-1.77(\mathrm{~m}, 1 \mathrm{H}), 1.64(\mathrm{~m}, 4 \mathrm{H}) ;{ }^{13} \mathrm{C}$ NMR $(125 \mathrm{MHz}$, $\left.\mathrm{CDCl}_{3}\right) \delta 153.90,153.45,142.31,130.78,130.72,119.57,119.50,117.77,103.28,68.81,68.77,65.20,65.06,58.04,41.53$, 39.47, 27.33, 23.67; IR (thin film, $\mathrm{cm}^{-1}$ ) 2935, 1801, 1767, 1450, 1350, 1326, 1303, 1242, 1212, 1158, 1121, 1034, 962; HRMS (ESI) $\mathrm{C}_{19} \mathrm{H}_{28} \mathrm{~N}_{2} \mathrm{O}_{8}$ Sna $(\mathrm{M}+\mathrm{Na})^{+}$467.1458, found $467.1451 \mathrm{~m} / \mathrm{z}$.

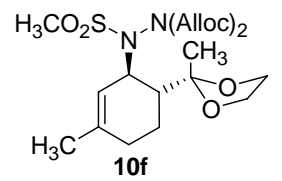

Acetal 10f: The reaction was run with $0.28 \mathrm{mmol} 8 \mathrm{ff}$. The product was isolated as a clear oil $(100 \mathrm{mg}$, 76\%). ${ }^{1} \mathrm{H}$ NMR (500 MHz, $\left.\mathrm{CDCl}_{3}\right) \delta 5.87-5.96(\mathrm{~m}, 2 \mathrm{H}), 5.45(\mathrm{~s}, 1 \mathrm{H}), 5.39-5.42(\mathrm{~m}, 2 \mathrm{H}), 5.26-5.28$ (m, 2H), $4.51-4.74(\mathrm{~m}, 4 \mathrm{H}), 4.51(\mathrm{~s}, 1 \mathrm{H}), 3.86-3.92(\mathrm{~m}, 4 \mathrm{H}), 3.71(\mathrm{~s}, 3 \mathrm{H}), 2.52(\mathrm{~m}, 1 \mathrm{H}), 1.92-1.95$ (m, 1H), $1.75-1.85(\mathrm{~m}, 2 \mathrm{H}), 1.64(\mathrm{~s}, 3 \mathrm{H}), 1.51-1.57(\mathrm{~m}, 1 \mathrm{H}), 1.23(\mathrm{~s}, 3 \mathrm{H}) ;{ }^{13} \mathrm{C}$ NMR $(125 \mathrm{MHz}$, $\left.\mathrm{CDCl}_{3}\right) \delta 153.77,152.98,141.52,130.81,130.73,119.52,119.19,117.45,111.20,68.70,68.49,65.01$, 63.87, 54.96, 43.20, 41.63, 25.94, 23.91; IR (thin film, $\mathrm{cm}^{-1}$ ) 2938, 2891, 1800, 1767, 1447, 1348, 1326, 1245, 1214, 1159, 1104, 1039, 949; HRMS (ESI) $\mathrm{C}_{20} \mathrm{H}_{30} \mathrm{~N}_{2} \mathrm{O}_{8} \mathrm{Sna}(\mathrm{M}+\mathrm{Na})^{+} 481.1615$, found $481.1606 \mathrm{~m} / \mathrm{z}$.

General procedure for Alloc deprotections: ${ }^{2}$ Acetal protected, Diels-Alder adduct 10 (1 equiv) and THF (0.2 M) were added to a flame dried, $10 \mathrm{~mL}$ round bottom flask under argon. Diethyl amine (15 equiv) was added via syringe, followed by tris(dibenzylideneacetone)dipalladium chloroform adduct $\left(\mathrm{Pd}_{2}\left(\mathrm{dba}_{3}\right)\right)(5 \mathrm{~mol} \%)$ and 1,2-bis(diphenylphosphino)ethane (dppe)

\footnotetext{
${ }^{2}$ Genêt, J. P.; Blart, E.; Savignac, M.; Lemeune, S.; Lemaire-Audoire, S.; Bernard, J. M. Synlett. 1993, 680-682.
} 
(10 mol\%), the reaction flask was capped with a yellow Teflon cap, and the mixture was stirred for 30 min. The solvent was removed by rotary evaporation and the residue was purified by flash column chromatography to provide deprotected hydrazine 11.
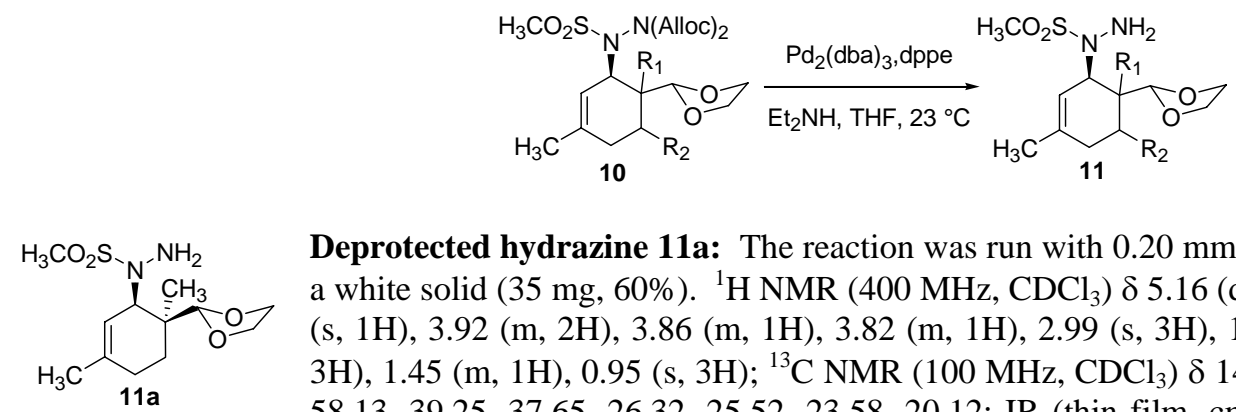

Deprotected hydrazine 11a: The reaction was run with $0.20 \mathrm{mmol}$ 10a. The product was isolated as a white solid (35 mg, 60\%). ${ }^{1} \mathrm{H}$ NMR $\left(400 \mathrm{MHz}, \mathrm{CDCl}_{3}\right) \delta 5.16(\mathrm{~d}, 1 \mathrm{H}, \mathrm{J}=2.8 \mathrm{~Hz}), 4.80(\mathrm{~s}, 1 \mathrm{H}), 4.37$ (s, 1H), $3.92(\mathrm{~m}, 2 \mathrm{H}), 3.86(\mathrm{~m}, 1 \mathrm{H}), 3.82(\mathrm{~m}, 1 \mathrm{H}), 2.99(\mathrm{~s}, 3 \mathrm{H}), 1.99(\mathrm{~m}, 2 \mathrm{H}), 1.85(\mathrm{~m}, 1 \mathrm{H}), 1.75(\mathrm{~s}$, 3H), 1.45 (m, 1H), 0.95 (s, 3H); ${ }^{13} \mathrm{C}$ NMR $\left(100 \mathrm{MHz}, \mathrm{CDCl}_{3}\right) \delta 143.05,116.24,107.19,65.35,64.83$, 58.13, 39.25, 37.65, 26.32, 25.52, 23.58, 20.12; IR (thin film, $\mathrm{cm}^{-1}$ ) 2935, 2360, 1319, 1143, 1096; HRMS (ESI) $\mathrm{C}_{12} \mathrm{H}_{22} \mathrm{~N}_{2} \mathrm{O}_{4} \mathrm{Sna}\left([\mathrm{M}+\mathrm{Na}]^{+}\right)$313.1192, found 313.1188.

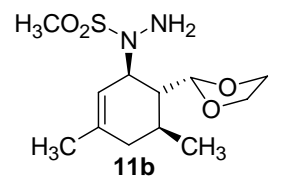

Deprotected hydrazine 11b: The reaction was run with $0.16 \mathrm{mmol}$ 10b. The product was isolated as a white solid (41 mg, 90\%). ${ }^{1} \mathrm{H}$ NMR $\left(500 \mathrm{MHz}, \mathrm{CDCl}_{3}\right) \delta 5.16(\mathrm{~s}, 1 \mathrm{H}), 5.08(\mathrm{~s}, 1 \mathrm{H}), 4.80(\mathrm{~d}, 1 \mathrm{H}, \mathrm{J}=$ $9.0 \mathrm{~Hz}), 3.97-4.02(\mathrm{~m}, 2 \mathrm{H}), 3.81-4.01(\mathrm{~m}, 2 \mathrm{H}), 3.53(\mathrm{~s}, 2 \mathrm{H}), 2.98(\mathrm{~s}, 3 \mathrm{H}), 1.87-1.95(\mathrm{~m}, 2 \mathrm{H}), 1.71-$ $1.79(\mathrm{~m}, 2 \mathrm{H}), 1.69$ (s, 3H), 1.03 (d, 3H, J = $7.0 \mathrm{~Hz}) ;{ }^{13} \mathrm{C}$ NMR $\left(125 \mathrm{MHz}, \mathrm{CDCl}_{3}\right) \delta 142.62,119.33$, 103.63, 64.93, 64.72, 55.98, 43.20, 39.46, 37.90, 29.85, 23.19, 19.89; IR (thin film, $\mathrm{cm}^{-1}$ ) 3360, 2892, 1309, 1156, 1126, 1033, 962, 918, 783; HRMS (ESI) $\mathrm{C}_{12} \mathrm{H}_{22} \mathrm{~N}_{2} \mathrm{O}_{4}$ Sna ([M+Na $]^{+}$) 313.1192, found 313.1184.

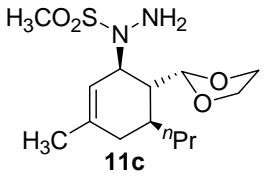

Deprotected hydrazine 11c: The reaction was run with $0.29 \mathrm{mmol} \mathrm{10c.} \mathrm{The} \mathrm{product} \mathrm{was} \mathrm{isolated} \mathrm{as}$ a white solid (59 mg, 65\%). ${ }^{1} \mathrm{H}$ NMR (500 MHz, $\left.\mathrm{CDCl}_{3}\right) \delta 0.86(\mathrm{t}, 3 \mathrm{H}, \mathrm{J}=7 \mathrm{~Hz}), 1.28-1.16(\mathrm{~m}, 2 \mathrm{H})$, $1.42-1.32(\mathrm{~m}, 1 \mathrm{H}), 1.62-1.54(\mathrm{~m}, 1 \mathrm{H}), 1.69(\mathrm{~s}, 3 \mathrm{H}), 1.76-1.65(\mathrm{~m}, 1 \mathrm{H}), 1.88-1.80(\mathrm{~m}, 2 \mathrm{H}), 1.98-1.93$ (m, 1H), 2.97 (s, 3H), 3.46 (s, br, 2H), 3.86-3.76 (m, 2H), 4.02-3.96 (m, 2H), 4.82-4.76 (m, 1H), 5.07 (s, 1H), $5.15(\mathrm{~s}, 1 \mathrm{H}) ;{ }^{13} \mathrm{C}$ NMR $\left(125 \mathrm{MHz}, \mathrm{CDCl}_{3}\right) \delta 14.35,19.05,23.34,34.16,35.15,35.44,37.90$, 41.35, 55.84, 64.81, 103.55, 119.17, 142.40; IR (thin film, $\mathrm{cm}^{-1}$ ) 3360, 2958, 2912, 1620, 1439, 1311, 1155, 1037; HRMS (ESI) $\mathrm{C}_{14} \mathrm{H}_{27} \mathrm{~N}_{2} \mathrm{O}_{4} \mathrm{~S}(\mathrm{M}+\mathrm{H})^{+}$319.1686, found $319.1672 \mathrm{~m} / \mathrm{z}$.

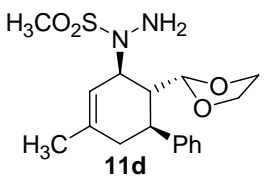

Deprotected hydrazine 11d: The reaction was run with $0.22 \mathrm{mmol} 10 \mathrm{~d}$. The product was isolated as a white solid (70 mg, 94\%). ${ }^{1} \mathrm{H}$ NMR (500 MHz, $\left.\mathrm{CDCl}_{3}\right) \delta 7.28-7.31(\mathrm{~m}, 2 \mathrm{H}), 7.19-7.26(\mathrm{~m}, 3 \mathrm{H})$, $5.30(\mathrm{~s}, 1 \mathrm{H}), 5.03(\mathrm{~s}, 1 \mathrm{H}, \mathrm{J}=9.0 \mathrm{~Hz}), 4.63(\mathrm{~s}, 1 \mathrm{H}), 3.85-3.88(\mathrm{~m}, 1 \mathrm{H}), 3.62-3.67$ (m, 4H), 3.52 (s, 2H), 3.01 (s, 3H), $2.42-2.46$ (m, 1H), $2.25-2.31$ (m, 1H), 2.10 (dd, 1H, J = 17.5 Hz, J = 4.5 Hz), 1.74 (s, 3H); ${ }^{13} \mathrm{C}$ NMR (125 MHz, $\left.\mathrm{CDCl}_{3}\right) \delta 143.47,141.92,128.45,127.78,126.48,119.94,103.36$, 64.83, 55.28, 42.30, 42.13, 39.30, 38.06, 23.07; IR (thin film, $\mathrm{cm}^{-1}$ ) 3359, 3027, 2895, 1311, 1152, 1030, 954; HRMS (ESI) $\mathrm{C}_{17} \mathrm{H}_{24} \mathrm{~N}_{2} \mathrm{O}_{4} \mathrm{~S}(\mathrm{M})^{+}$353.1529, found $153.1529 \mathrm{~m} / \mathrm{z}$.

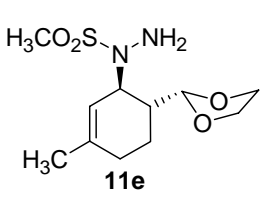

Deprotected hydrazine 11e: The reaction was run with $0.11 \mathrm{mmol}$ 10e. The product was isolated as a white solid (20 mg, 65\%). ${ }^{1} \mathrm{H}$ NMR (500 MHz, $\left.\mathrm{CDCl}_{3}\right) \delta 5.21(\mathrm{~s}, 1 \mathrm{H}), 4.92(\mathrm{~d}, 1 \mathrm{H}, \mathrm{J}=3.0 \mathrm{~Hz}), 4.70$ (d, $1 \mathrm{H}, \mathrm{J}=14 \mathrm{~Hz}$ ), $3.82-3.99$ (m, 4H), 3.32 (s, 2H), 3.00 (s, 3H), $1.88-2.02$ (m, 4H), $1.71(\mathrm{~s}, 3 \mathrm{H})$, 1.52 - $1.58(\mathrm{~m}, 1 \mathrm{H}) ;{ }^{13} \mathrm{C}$ NMR $\left(125 \mathrm{MHz}, \mathrm{CDCl}_{3}\right) \delta 143.33,119.65,104.32$, 65.38, 65.02, 56.69, 39.01, 37.84, 29.35, 23.39, 21.42; IR (thin film, $\mathrm{cm}^{-1}$ ) 3368, 2928, 2896, 1307, 1152, 1035, 957; HRMS (ESI) $\mathrm{C}_{11} \mathrm{H}_{20} \mathrm{~N}_{2} \mathrm{O}_{4} \mathrm{Sna}(\mathrm{M}+\mathrm{Na})^{+}$299.1036, found $299.1030 \mathrm{~m} / \mathrm{z}$.

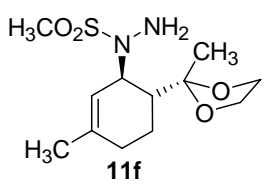

Deprotected hydrazine 11f: The reaction was run with $0.22 \mathrm{mmol}$ 10a. The product was isolated as a white solid (52 mg, 82\%). ${ }^{1} \mathrm{H}$ NMR (500 MHz, $\left.\mathrm{CDCl}_{3}\right) \delta 5.19(\mathrm{~s}, 1 \mathrm{H}), 4.71$ (d, $\left.1 \mathrm{H}, \mathrm{J}=9.0 \mathrm{~Hz}\right), 3.96$ (m, 1H), 3.88 (m, 3H), 3.57 (s, 2H), 2.99 (s, 3H), $1.90-2.08$ (m, 3H), 1.82 (m, 1H), 1.66 (s, 3H), 1.45 
(m, 1H), 1.26 (s, 3H); ${ }^{13} \mathrm{C}$ NMR (125 MHz, $\left.\mathrm{CDCl}_{3}\right) \delta 142.25,121.21,110.94,64.90,63.75,56.61,43.97,38.21,30.04$, 24.02, 22.97, 19.83; IR (thin film, $\mathrm{cm}^{-1}$ ) 3361, 2967, 2934, 2891, 1445, 1379, 1308, 1199, 1153, 1050, 958; HRMS (ESI) $\mathrm{C}_{12} \mathrm{H}_{22} \mathrm{~N}_{2} \mathrm{O}_{4} \mathrm{Sna}(\mathrm{M}+\mathrm{Na})^{+} 313.1192$, found $313.1185 \mathrm{~m} / \mathrm{z}$.
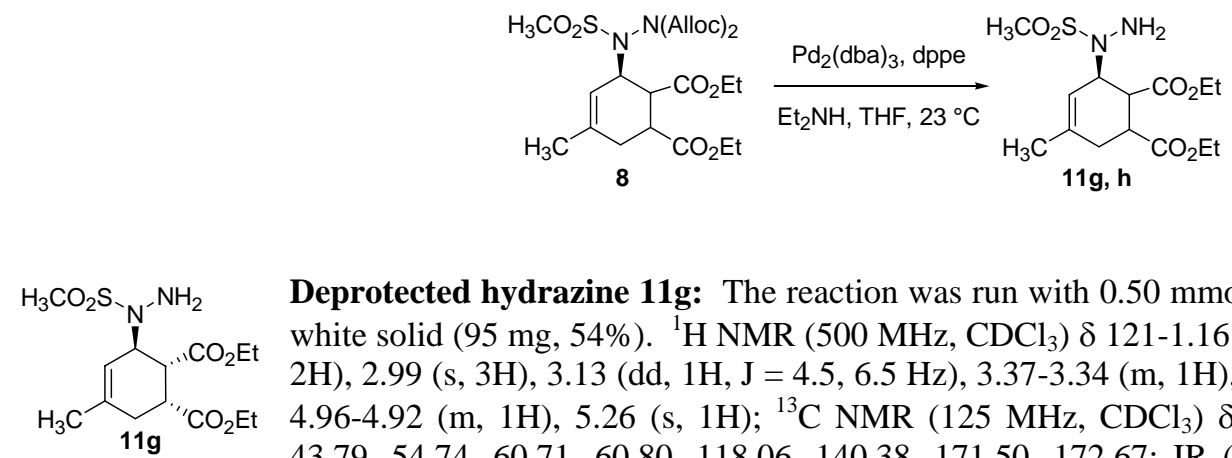

Deprotected hydrazine 11g: The reaction was run with $0.50 \mathrm{mmol} \mathbf{8 g}$. The product was isolated as a white solid (95 mg, 54\%). ${ }^{1} \mathrm{H}$ NMR (500 MHz, $\left.\mathrm{CDCl}_{3}\right) \delta 121-1.16(\mathrm{~m}, 6 \mathrm{H}), 1.71(\mathrm{~s}, 3 \mathrm{H}), 2.37-2.25$ (m, $2 \mathrm{H}), 2.99$ (s, 3H), 3.13 (dd, $1 \mathrm{H}, \mathrm{J}=4.5,6.5 \mathrm{~Hz}), 3.37-3.34(\mathrm{~m}, 1 \mathrm{H}), 3.58$ (s, br, 2H), 4.16-4.02 (m, 4H), 4.96-4.92 (m, 1H), $5.26(\mathrm{~s}, 1 \mathrm{H}) ;{ }^{13} \mathrm{C}$ NMR $\left(125 \mathrm{MHz}, \mathrm{CDCl}_{3}\right) \delta$ 13.98, 23.46, 30.25, 37.82, 39.94, 43.79, 54.74, 60.71, 60.80, 118.06, 140.38, 171.50, 172.67; IR (thin film, $\mathrm{cm}^{-1}$ ) 3367, 2981, 2936, 2360, 2341, 1733, 1320, 1197, 1151; HRMS (ESI) $\mathrm{C}_{14} \mathrm{H}_{25} \mathrm{~N}_{2} \mathrm{O}_{6} \mathrm{~S}(\mathrm{M}+\mathrm{H})^{+} 349.1428$, found $349.1416 \mathrm{~m} / \mathrm{z}$.

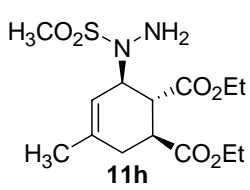

Deprotected hydrazine 11h: The reaction was run with $0.38 \mathrm{mmol} \mathbf{8 f}$. The product was isolated as a white solid (38 mg, 28\%). ${ }^{1} \mathrm{H}$ NMR (300 MHz, $\left.\mathrm{CDCl}_{3}\right) \delta 1.25-1.20(\mathrm{~m}, 6 \mathrm{H}), 1.72(\mathrm{~s}, 3 \mathrm{H}), 2.20-2.07$ (m, 1H), 2.32 (dd, $1 \mathrm{H}, \mathrm{J}=5.4,17 \mathrm{~Hz}$ ), 2.86 (dd, $1 \mathrm{H}, \mathrm{J}=11,12 \mathrm{~Hz}$ ), 2.96 (s, 3H), 3.06 (ddd, $1 \mathrm{H}, \mathrm{J}=5.4$, 12, $12 \mathrm{~Hz}), 3.67$ (s, 2H), 4.22-4.09 (m, 4H), 4.88-4.80 (m, 1H), 5.31 (s, br, $1 \mathrm{H}) ;{ }^{13} \mathrm{C}$ NMR $(75 \mathrm{MHz}$, $\left.\mathrm{CDCl}_{3}\right) \delta 14.08,14.16,22.84,32.49,38.18,41.73,44.74,58.23,60.97,61.02,120.04,140.04,172.61$, 173.15; IR (thin film, $\mathrm{cm}^{-1}$ ) 3369, 2981, 2936, 1732, 1335, 1188, 1155; HRMS (ESI) $\mathrm{C}_{14} \mathrm{H}_{24} \mathrm{~N}_{2} \mathrm{O}_{6} \mathrm{Sna}(\mathrm{M}+\mathrm{Na})^{+}$371.1247, found $371.1233 \mathrm{~m} / \mathrm{z}$.

General procedure for the elimination/rearrangement of deprotected hydrazine (11): Deprotected hydrazine 11 (1 equiv), tetrabutylammonium acetate (4 equiv), and THF $(0.2 \mathrm{mmol}$ ) were added to a $15 \mathrm{~mL}$ round-bottom flask equipped with a reflux condenser. The reaction was stirred for $18 \mathrm{~h}$ at $40{ }^{\circ} \mathrm{C}$. The mixture was then allowed to cool to ambient temperature and diluted with $10 \%$ diethyl ether/pentane. The organics were washed with $2 \mathrm{~mL}_{2} \mathrm{O}$ and the combined organics were dried with $\mathrm{Na}_{2} \mathrm{SO}_{4}$ and removed by rotary evaporation. The product was purified by flash column chromatography (1:9 diethyl ether/pentanes) to provide pure cyclohexene $\mathbf{1 2}$.
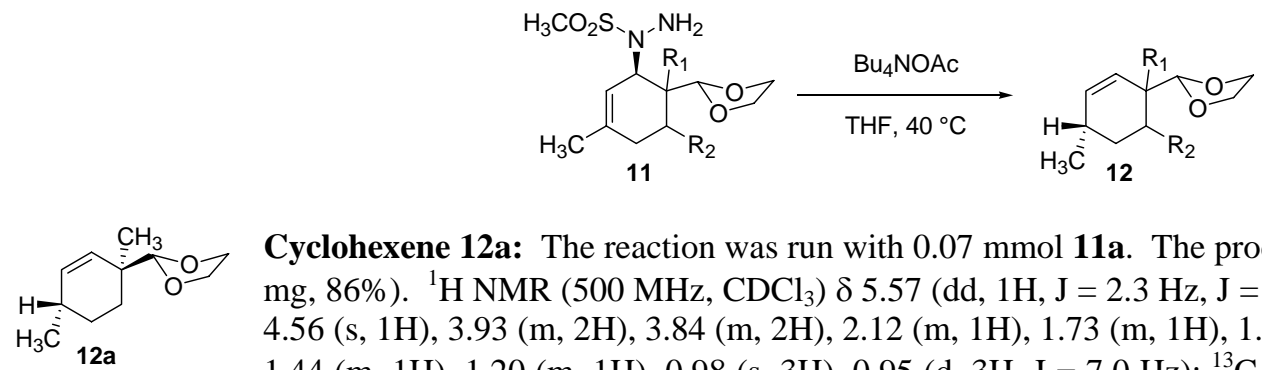

Cyclohexene 12a: The reaction was run with $0.07 \mathrm{mmol} \mathrm{11a}$. The product was isolated as a clear oil (11.2 mg, 86\%). ${ }^{1} \mathrm{H}$ NMR (500 MHz, $\left.\mathrm{CDCl}_{3}\right) \delta 5.57(\mathrm{dd}, 1 \mathrm{H}, \mathrm{J}=2.3 \mathrm{~Hz}, \mathrm{~J}=10.0 \mathrm{~Hz}), 5.46$ (d, 2H, J = $\left.10.5 \mathrm{~Hz}\right)$, $4.56(\mathrm{~s}, 1 \mathrm{H}), 3.93(\mathrm{~m}, 2 \mathrm{H}), 3.84(\mathrm{~m}, 2 \mathrm{H}), 2.12(\mathrm{~m}, 1 \mathrm{H}), 1.73(\mathrm{~m}, 1 \mathrm{H}), 1.63(\mathrm{dt}, 1 \mathrm{H}, \mathrm{J}=3.5 \mathrm{~Hz}, \mathrm{~J}=13.0 \mathrm{~Hz})$, $1.44(\mathrm{~m}, 1 \mathrm{H}), 1.20(\mathrm{~m}, 1 \mathrm{H}), 0.98(\mathrm{~s}, 3 \mathrm{H}), 0.95(\mathrm{~d}, 3 \mathrm{H}, \mathrm{J}=7.0 \mathrm{~Hz}) ;{ }^{13} \mathrm{C}$ NMR $\left(125 \mathrm{MHz}, \mathrm{CDCl}_{3}\right) \delta 134.37$, 130.51, 109.52, 65.31, 65.26, 39.02, 30.61, 29.69, 29.50, 27.60, 21.64; IR (thin film, $\mathrm{cm}^{-1}$ ) 2947, 2924, 2850, 2358, 2336, 1456, 1098.

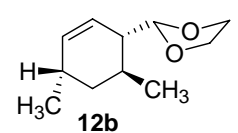

Cyclohexene 12b: The reaction was run with $0.14 \mathrm{mmol}$ 11b. The product was isolated as a clear oil (23 mg, 92\%). ${ }^{1} \mathrm{H}$ NMR (500 MHz, $\left.\mathrm{CDCl}_{3}\right) \delta 5.70(\mathrm{~d}, 1 \mathrm{H}, \mathrm{J}=10.0 \mathrm{~Hz}), 5.55(\mathrm{~d}, 1 \mathrm{H}, \mathrm{J}=10.0 \mathrm{~Hz}), 4.78(\mathrm{~d}, 1 \mathrm{H}$, $\mathrm{J}=4.5 \mathrm{~Hz}), 3.94(\mathrm{~m}, 2 \mathrm{H}), 3.82(\mathrm{~m}, 2 \mathrm{H}), 2.19(\mathrm{~m}, 1 \mathrm{H}), 1.94(\mathrm{~m}, 2 \mathrm{H}), 1.46(\mathrm{~m}, 1 \mathrm{H}), 1.36(\mathrm{~m}, 1 \mathrm{H}), 0.96-$ 1.02 (m, 6H); ${ }^{13} \mathrm{C}$ NMR (125 MHz, CDCl $) \delta 135.15,123.53,106.01,65.01,64.79,45.61,35.97,26.99$, 26.15, 21.29, 20.07; IR (thin film, $\mathrm{cm}^{-1}$ ) 3024, 2956, 2926, 2873, 1458, 1405, 1376, 1146, 1123, 1057, 1037, 966. 


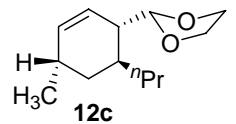

Cyclohexene 12c: The reaction was run with $0.14 \mathrm{mmol} 11 \mathrm{c}$. The product was isolated as a clear oil (26 mg, 87\%). ${ }^{1} \mathrm{H}$ NMR $\left(500 \mathrm{MHz}, \mathrm{CDCl}_{3}\right) \delta 0.87$ (t, 3H, J = $\left.6.5 \mathrm{~Hz}\right), 0.95$ (d, 3H, J =7 Hz), 1.37-1.25 (m, $5 \mathrm{H}), 1.55-1.49(\mathrm{~m}, 1 \mathrm{H}), 1.87-1.82(\mathrm{~m}, 1 \mathrm{H}), 2.02-1.98(\mathrm{~m}, 1 \mathrm{H}), 2.17-2.09(\mathrm{~m}, 1 \mathrm{H}), 3.85-3.81(\mathrm{~m}, 2 \mathrm{H}), 4.00-$ $3.90(\mathrm{~m}, 2 \mathrm{H}), 4.75(\mathrm{~d}, 1 \mathrm{H}, \mathrm{J}=5.5 \mathrm{~Hz}), 5.57-5.53(\mathrm{~m}, 1 \mathrm{H}), 5.71-5.66(\mathrm{~m}, 1 \mathrm{H}) ;{ }^{13} \mathrm{C}$ NMR $\left(125 \mathrm{MHz}, \mathrm{CDCl}_{3}\right) \delta$ 14.24, 20.25, 21.38, 26.27, 31.61, 32.61, 35.53, 43.78, 64.68, 65.03, 106.36, 123.71, 123.78, 135.52, 135.58; IR (thin film, $\mathrm{cm}^{-1}$ ) 2955, 2926, 2871, 1453, 1123, 1053.

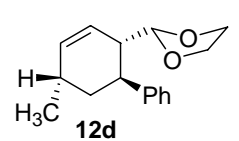

Cyclohexene 12d: The reaction was run with $0.17 \mathrm{mmol}$ 11d. The product was isolated as a clear oil (31 mg, 81\%). ${ }^{1} \mathrm{H}$ NMR (500 MHz, $\left.\mathrm{CDCl}_{3}\right) \delta 7.32-7.18(\mathrm{~m}, 5 \mathrm{H}), 5.88-5.83(\mathrm{~m}, 1 \mathrm{H}), 5.80-5.76(\mathrm{~m}, 1 \mathrm{H})$, $4.68(\mathrm{~d}, 1 \mathrm{H}, \mathrm{J}=4.0 \mathrm{~Hz}$ ), $3.98-3.89(\mathrm{~m}, 2 \mathrm{H}), 3.82-3.75(\mathrm{~m}, 2 \mathrm{H}), 2.98$ (ddd, $1 \mathrm{H}, \mathrm{J}=3.5 \mathrm{~Hz}, \mathrm{~J}=8.0 \mathrm{~Hz}, \mathrm{~J}$ $=11.0 \mathrm{~Hz}), 2.69-2.63(\mathrm{~m}, 1 \mathrm{H}), 2.21(\mathrm{~s}, 1 \mathrm{H}), 1.96-1.88(\mathrm{~m}, 1 \mathrm{H}), 1.64-1.57(\mathrm{~m}, 1 \mathrm{H}), 1.05(\mathrm{~d}, 3 \mathrm{H}, \mathrm{J}=7.0$ $\mathrm{Hz}) ;{ }^{13} \mathrm{C}$ NMR $\left(125 \mathrm{MHz}, \mathrm{CDCl}_{3}\right) \delta 145.55,135.36,128.32,127.58,126.06,123.92,104.69,65.23,64.92,44.31,37.89$, 37.29, 28.35, 21.05; IR (thin film, $\mathrm{cm}^{-1}$ ) 3026, 2956, 2923, 2872, 1603, 1494, 1453, 1404, 1366, 1149, 1134, 1053, 1038, 945, 756, 701.

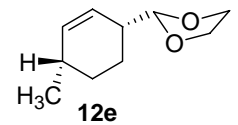

Cyclohexene 12e: The reaction was run with $0.08 \mathrm{mmol}$ 11e. The product was isolated as a clear oil (9 mg, 94\%). ${ }^{1} \mathrm{H}$ NMR (500 MHz, $\left.\mathrm{CDCl}_{3}\right) \delta 5.72(\mathrm{~d}, 1 \mathrm{H}, \mathrm{J}=10.0 \mathrm{~Hz}), 5.61(\mathrm{~d}, 1 \mathrm{H}, \mathrm{J}=10.0 \mathrm{~Hz}), 4.68(\mathrm{~d}, 1 \mathrm{H}$, $\mathrm{J}=6.0 \mathrm{~Hz}), 3.91-3.99(\mathrm{~m}, 2 \mathrm{H}), 3.84(\mathrm{~m}, 2 \mathrm{H}), 2.26(\mathrm{~m}, 1 \mathrm{H}), 2.16(\mathrm{~m}, 1 \mathrm{H}), 1.67(\mathrm{~m}, 3 \mathrm{H}), 1.38(\mathrm{~m}, 1 \mathrm{H})$, 0.97 (d, 3H, J = 7.0 Hz); ${ }^{13} \mathrm{C}$ NMR $\left(125 \mathrm{MHz}, \mathrm{CDCl}_{3}\right) \delta 135.94,124.83,106.70,65.04,64.83,39.15,29.52$, 28.19, 21.24; IR (thin film, $\mathrm{cm}^{-1}$ ) 3023, 2955, 2927, 2870, 1454, 1393, 1148, 1118, 1082.

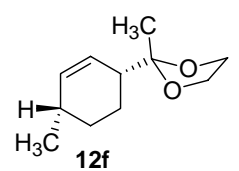

Cyclohexene 12f: The reaction was run with $0.15 \mathrm{mmol}$ 11f. The product was isolated as a clear oil (25 mg, 91\%). ${ }^{1} \mathrm{H}$ NMR $\left(500 \mathrm{MHz}, \mathrm{CDCl}_{3}\right) \delta 5.70(\mathrm{~m}, 1 \mathrm{H}), 5.62(\mathrm{~d}, 1 \mathrm{H}, \mathrm{J}=10.0 \mathrm{~Hz}), 3.93(\mathrm{~m}, 4 \mathrm{H}), 2.29(\mathrm{~m}$, $1 \mathrm{H}), 2.15$ (m, 1H), $1.66(\mathrm{~m}, 2 \mathrm{H}), 1.57(\mathrm{~m}, 1 \mathrm{H}), 1.45$ (m, 1H), 1.24 (s, 3H), 0.97 (d, 3H, J = $7.5 \mathrm{~Hz}) ;{ }^{13} \mathrm{C}$ NMR (125 MHz, $\left.\mathrm{CDCl}_{3}\right) \delta 135.06,126.03,111.71,64.66,64.62,43.76,28.90,28.58,21.13,21.05,20.50$; IR (thin film, $\mathrm{cm}^{-1}$ ) 3022, 2955, 2871, 1456, 1378, 1210, 1150, 1048, 947.

\section{Elimination/rearrangement to form cyclohexene 13}
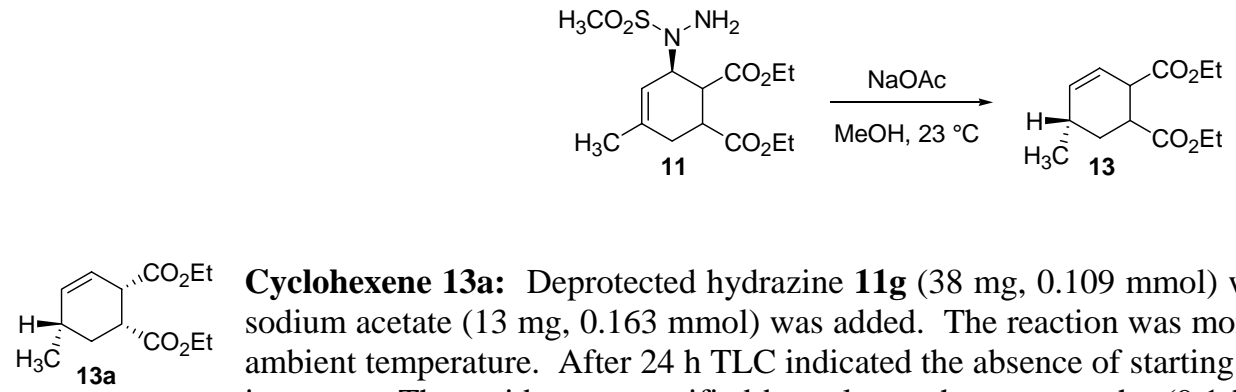

Cyclohexene 13a: Deprotected hydrazine $11 \mathrm{~g}$ (38 $\mathrm{mg}, 0.109 \mathrm{mmol})$ was dissolved in $\mathrm{MeOH}(0.7 \mathrm{~mL})$ and sodium acetate (13 mg, $0.163 \mathrm{mmol}$ ) was added. The reaction was monitored by TLC and stirred for $24 \mathrm{~h}$ at ambient temperature. After $24 \mathrm{~h}$ TLC indicated the absence of starting material and the $\mathrm{MeOH}$ was removed in vacuo. The residue was purified by column chromatography (9:1 hexanes/ethyl acetate) to yield $24 \mathrm{mg}$ (91\%) of 13a as a clear oil. ${ }^{1} \mathrm{H}$ NMR $\left(500 \mathrm{MHz}, \mathrm{CDCl}_{3}\right) \delta 1.01(\mathrm{~d}, 3 \mathrm{H}, \mathrm{J}=6.5 \mathrm{~Hz}), 1.23-1.19(\mathrm{~m}, 6 \mathrm{H}), 1.68-159(\mathrm{~m}, 1 \mathrm{H})$, 2.24-2.17 (m, 2H), 2.59 (ddd, 1H, J = 2.5, 5.5, 12. $5 \mathrm{~Hz}$ ), 3.51-3.47 (m, 1H), 4.25-4.03 (m, 4H), 5.73-5.65 (m, 2H); ${ }^{13} \mathrm{C}$ NMR $\left(125 \mathrm{MHz}, \mathrm{CDCl}_{3}\right) \delta 14.11,21.04,29.44,31.09,41.24,42.15,60.42,60.59,122.23,136.54,172.04,173.42$; IR (thin film, $\mathrm{cm}^{-1}$ ) 2960, 2873, 1741, 1263, 1191; HRMS (ESI) $\mathrm{C}_{13} \mathrm{H}_{20} \mathrm{O}_{4} \mathrm{Na}(\mathrm{M}+\mathrm{Na})^{+} 263.1254$, found $263.1255 \mathrm{~m} / \mathrm{z}$. 
The following selected nOe was observed for cyclohexene 13a:
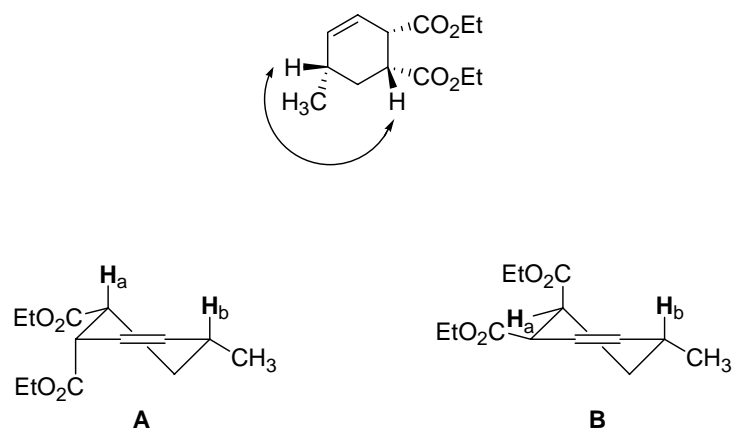

The observed nOe between $\mathrm{H}_{\mathrm{a}}$ and $\mathrm{H}_{\mathrm{b}}$ is consistent with structure $\mathbf{A}$ as $\mathrm{H}_{\mathrm{a}}$ and $\mathrm{H}_{\mathrm{b}}$ are both pseudoaxial and in close proximity. However, no nOe would be observed between $\mathrm{H}_{\mathrm{a}}$ and $\mathrm{H}_{\mathrm{b}}$ for diasteromer $\mathbf{B}$.

".

Cyclohexene 13b: Deprotected hydrazine 11h $(25 \mathrm{mg}, 0.07 \mathrm{mmol})$ was dissolved in $\mathrm{MeOH}(0.46 \mathrm{~mL})$ and sodium acetate ( $8.8 \mathrm{mg}, 0.105 \mathrm{mmol}$ ) was added. The reaction was monitored by TLC and stirred for $24 \mathrm{~h}$ at ambient temperature. After $24 \mathrm{~h}$ TLC indicated the absence of starting material and the $\mathrm{MeOH}$ was removed in vacuo. The residue was purified by column chromatography (9:1 hexanes/ethyl acetate) to yield $8 \mathrm{mg}$ (48\%) of $\mathbf{1 3 b}$ as a clear oil. ${ }^{1} \mathrm{H}$ NMR (500 MHz, $\left.\mathrm{CDCl}_{3}\right) \delta 1.02(\mathrm{~d}, 3 \mathrm{H}, \mathrm{J}=7 \mathrm{~Hz}), 1.24(\mathrm{t}, 6 \mathrm{H}, \mathrm{J}=7 \mathrm{~Hz}), 1.68$ (ddd, app dt, $1 \mathrm{H}, \mathrm{J}=4,4,13 \mathrm{~Hz}$ ), 1.91 (ddd, 1H, J = 6, 10.5, $13.5 \mathrm{~Hz}$ ), 2.31-2.24 (m, 1H), 3.00 (ddd, $1 \mathrm{H}, \mathrm{J}=3.5,8,11$ ) Hz), $3.49-3.45$ (m, 1H), 4.14 (q, 4H, J = 7.5 Hz), 5.73-5.66 (m, 2H); ${ }^{13} \mathrm{C}$ NMR (125 MHz, CDCl $\left.{ }_{3}\right) \delta 14.13,14.19,20.56,28.04,31.19$, 38.11, 43.38, 60.65, 60.90, 122.19, 134.38, 173.01, 174.87; IR (thin film, $\mathrm{cm}^{-1}$ ) 2960, 2873, 1733, 1195, 1161.

The following selected nOe was observed for cyclohexene 13b:

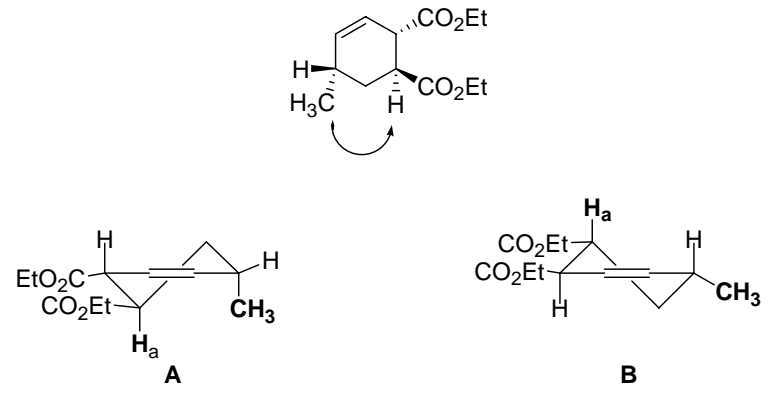

The observed nOe between methyne proton $\mathrm{H}_{\mathrm{a}}$ and the methyl group is consistent with structure $\mathbf{A}$ as both substituents are pseudoaxial and are in close proximity. This nOe is not consistent with diastereomer $\mathbf{B}$ as the two groups are on opposite sides of the ring.

\section{Synthesis of trans-fused decalin 16}<smiles>O=CC1CCCCC1</smiles><smiles>C=CCOC(=O)NN</smiles><smiles>CNN=Cc1ccccc1</smiles> 
Mono-Alloc hydrazone (S5): Allyloxycarbazate S3 (3.91, $33.7 \mathrm{mmol}$ ), aldehyde ${ }^{3}$ (5 g, $40 \mathrm{mmol}$ ), and THF (200 mL) were added to a $500 \mathrm{~mL}$ round-bottom flask and the reaction mixture was stirred for $3 \mathrm{~h}$. The solvent was removed by rotary evaporation and the residual oil was purified by column chromatography to yield $5.2 \mathrm{~g} \mathrm{(71 \% )}$ of S-5 as a white solid. ${ }^{1} \mathrm{H}$ NMR (400 MHz, CDCl $)_{3} \delta 1.52-1.62(\mathrm{~m}, 6 \mathrm{H}), 2.16-2.21(\mathrm{~m}, 2 \mathrm{H}), 2.26-2.32(\mathrm{~m}, 2 \mathrm{H}), 4.68$ (s, br, 2H), 5.22 (dd, $1 \mathrm{H}, \mathrm{J}=1$, $10 \mathrm{~Hz}$ ), 5.32 (d, 1H, J = $16 \mathrm{~Hz}$ ), $5.93(\mathrm{~s}, \mathrm{br}, 1 \mathrm{H}), 6.00$ (d, 1H, J = $10 \mathrm{~Hz}), 7.79$ (s, br, 1H), 7.91 (s, br, $1 \mathrm{H}) ;{ }^{13} \mathrm{C}$ NMR $(100$ $\mathrm{MHz}, \mathrm{CDCl}_{3}$ ) $\delta$ 26.19, 27.60, 28.14, 19.38, 37.27, 65.66, 66.27, 117.82, 118.62, 132.15, 143.85, 151.83, 153.40; IR (thin film, $\mathrm{cm}^{-1}$ ) 3229, 2931, 2854, 1718, 1648, 1543, 1247; HRMS (ESI) $\mathrm{C}_{12} \mathrm{H}_{19} \mathrm{~N}_{2} \mathrm{O}_{2}(\mathrm{M}+\mathrm{H})^{+}$223.1441, found $223.1448 \mathrm{~m} / \mathrm{z}$.

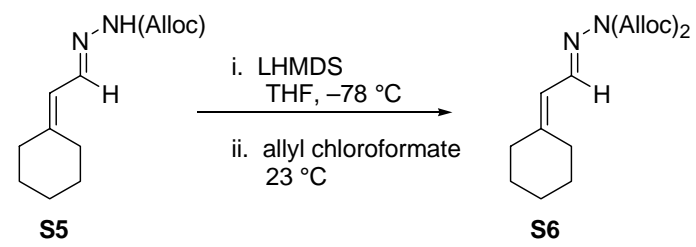

Bis-Alloc Hydrazone S6: Mono Alloc hydrazone S5 (1.0 g, $4.5 \mathrm{mmol})$ and THF (22 mL) were added to a flame dried 250 $\mathrm{mL}$ round-bottom flask under argon. The mixture was cooled to $-78{ }^{\circ} \mathrm{C}$ and LHMDS (1 M solution in hexanes, $4.5 \mathrm{~mL}, 4.5$ mmol) was added dropwise over $5 \mathrm{~min}$. The reaction was then stirred for an additional $30 \mathrm{~min}$ at $-78{ }^{\circ} \mathrm{C}$. Allylchloroformate $(0.5 \mathrm{~mL}, 4.72 \mathrm{mmol})$ was added via syringe and the reaction mixture was allowed to warm to ambient temperature and stirred for an additional $1.5 \mathrm{~h}$. The reaction mixture was diluted with diethyl ether $(50 \mathrm{~mL})$ and then washed with saturated $\mathrm{NaHCO}_{3}$ (2x20 mL). The organics were dried with $\mathrm{Na}_{2} \mathrm{SO}_{4}$ and the solvent was removed by rotary evaporation to provide a yellow oil. The residue was purified by flash chromatography (2:1 hexanes/ethyl acetate) to afford pure bisAlloc hydrazone $\mathbf{S 6}$ as a clear oil (1.3 g, 94). ${ }^{1} \mathrm{H}$ NMR (500 MHz, $\left.\mathrm{CDCl}_{3}\right) \delta 1.54-64(\mathrm{~m}, 6 \mathrm{H}), 2.20-2.25$ (m, 2H), 2.30-2.34 (m, 2H), 4.68-4.72 (m, 4H), 5.19-5.23 (m, 2H), 5.33 (dd, 2H, J = 1.5, $16.5 \mathrm{~Hz}$ ), 5.86-5.94 (m, 2H), 6.10 (d, 1H, J = $10 \mathrm{~Hz}), 8.22$ (dd, 1H, J = 1.2, $10 \mathrm{~Hz}$ ); ${ }^{13} \mathrm{C}$ NMR $\left(125 \mathrm{MHz}, \mathrm{CDCl}_{3}\right) \delta$ 26.13, 27.74, 28.13, 29.89, 37.75, 67.66, 118.14, 118.82, 131.16, 151.45, 159.83, 164.12; IR (thin film, $\mathrm{cm}^{-1}$ ) 2934, 2857, 1790, 1750, 1646, 1262, 1109; HRMS (ESI) $\mathrm{C}_{16} \mathrm{H}_{23} \mathrm{~N}_{2} \mathrm{O}_{4}(\mathrm{M}+\mathrm{H})^{+}$307.1652, found 307.1648 $\mathrm{m} / \mathrm{z}$.

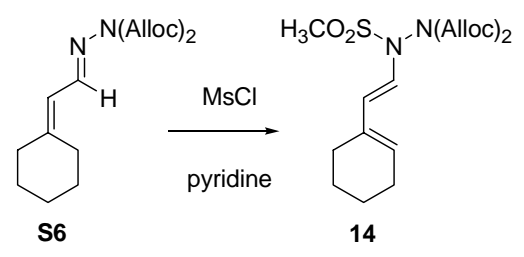

Synthesis of 14: Bis-Alloc hydrazone $\mathbf{S 6}(570 \mathrm{mg}, 1.86 \mathrm{mmol})$ and pyridine $(0.75 \mathrm{~mL}, 9.3 \mathrm{mmol})$ were added to a flamedried $10 \mathrm{~mL}$ round-bottom flask. Methanesulfonic chloride $(0.43 \mathrm{~mL}, 5.5 \mathrm{mmol})$ was added dropwise over $1 \mathrm{~min}$ and the reaction was sealed and stirred at ambient temperature for $2 \mathrm{~h}$. The reaction was diluted with methylene chloride and washed with $10 \% \mathrm{NH}_{4} \mathrm{Cl}(1 \times 10 \mathrm{~mL}), 10 \% \mathrm{NaHCO}_{3}(1 \times 10 \mathrm{~mL})$, and $\mathrm{H}_{2} \mathrm{O}(1 \times 10 \mathrm{~mL})$. The combined organics were dried with $\mathrm{Na}_{2} \mathrm{SO}_{4}$ and the solvent was removed by rotary evaporation to provide an orange oil. The residue was purified by flash chromatography (85:15 hexanes/ethyl acetate) to provide pure 14 as a clear oil $(1.04 \mathrm{~g}, 78 \%) .{ }^{1} \mathrm{H}$ NMR $\left(500 \mathrm{MHz}, \mathrm{CDCl}_{3}\right) \delta$ 1.59-1.52 (m, 2H), 1.69-1.62 (m, 2H), 2.11-2.05 (m, 4H), 3.14 (s, 3H), 4.81-4.72 (m, 4H), 5.28 (dd, 2H, J = 1, 10 Hz), 5.41 (dd, $2 \mathrm{H}, \mathrm{J}=1.5,17), 5.56(\mathrm{~d}, 1 \mathrm{H}, \mathrm{J}=14 \mathrm{~Hz}), 5.63(\mathrm{~s}, 1 \mathrm{H}), 5.96-5.87(\mathrm{~m}, 2 \mathrm{H}), 6.52(\mathrm{~d}, 1 \mathrm{H}, \mathrm{J}=14) ;{ }^{13} \mathrm{C}$ NMR $(100 \mathrm{MHz}$, $\mathrm{CDCl}_{3}$ ) $\delta$ 22.06, 22.14, 24.46, 25.58, 41.08, 68.58, 115.69, 119.14, 120.18, 128.43, 130.42, 132.25, 151.11; IR (thin film, $\mathrm{cm}^{-}$ $\left.{ }^{1}\right)$ 2943, 1806, 1775, 1645, 1365, 1245, 1164, 1110; HRMS (ESI) $\mathrm{C}_{17} \mathrm{H}_{25} \mathrm{~N}_{2} \mathrm{O}_{6} \mathrm{~S}(\mathrm{M}+\mathrm{H})^{+}$385.1428, found $385.1412 \mathrm{~m} / \mathrm{z}$.

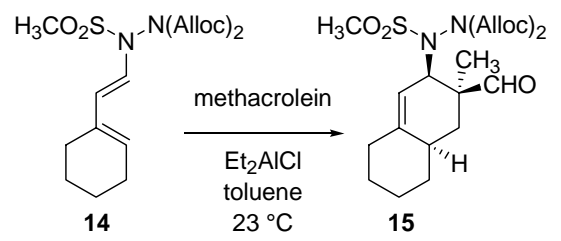

\footnotetext{
${ }^{3}$ Dauben, W. G.; Michno, D. M. J. Org. Chem. 1977, 42, 682.
} 
Synthesis of 15: $4 \AA$-Molecular sieves $(150 \mathrm{mg} / \mathrm{mmol})$ were added to a $25 \mathrm{~mL}$ round bottom flask and the system was flame dried under vacuum and charged with argon when cool. Toluene $(2.2 \mathrm{~mL})$ was added followed by 1-hydrazinodiene 14 (245 $\mathrm{mg}, 0.64 \mathrm{mmol}$ ) as a $1 \mathrm{~mL}$ solution in toluene. Diethylaluminum chloride (1.8 M solution in toluene, $0.14 \mathrm{~mL}, 0.25 \mathrm{mmol})$ was added to the reaction followed by methacrolein $(0.16 \mathrm{~mL}, 1.9 \mathrm{mmol})$. The reaction was stirred under argon for $24 \mathrm{~h}$ at ambient temperature and then purified by column chromatography without aqueous work-up to provide $135 \mathrm{mg}$ (50\%) of clear oil $\mathbf{1 5}$ as a 7.3:1 mixture of diastereomers. The relative stereochemistry of the major diasteromer was determined by analogy with Diels-Alder adduct 8a. ${ }^{1} \mathrm{H}$ NMR analysis of the crude reaction mixture reveals the aldehyde corresponding to the major endo-diasteromer is downfield from the exo-diasteromer An analytical sample was obtained after 3 chromatography columns. ${ }^{1} \mathrm{H}$ NMR $\left(500 \mathrm{MHz}, \mathrm{CDCl}_{3}\right) \delta 0.98(\mathrm{~s}, 3 \mathrm{H}), 1.22-1.08(\mathrm{~m}, 2 \mathrm{H}), 1.40-1.28(\mathrm{~m}, 2 \mathrm{H}), 1.89-1.68$ (m, $4 \mathrm{H}), 2.02-1.90(\mathrm{~m}, 2 \mathrm{H}), 2.26-2.19(\mathrm{~m}, 1 \mathrm{H}), 3.26(\mathrm{~s}, 3 \mathrm{H}), 4.43-4.40(\mathrm{~m}, 1 \mathrm{H}), 4.54$ (dddd, app ddt, $\mathrm{J}=1,1,6,13 \mathrm{~Hz}, 1 \mathrm{H})$, 4.64 (dddd, app ddt, J = 1, 1, 6, $13 \mathrm{~Hz}, 1 \mathrm{H}), 4.76-4.70$ (m, 2H), 5.30-5.24 (m, 3H), 5.39-5.35 (m, 1H), 5.45-5.40 (m, 1H), 5.96-5.85 (m, 2H), 9.94 (s, 1H); ${ }^{13} \mathrm{C}$ NMR (125 MHz, $\left.\mathrm{CDCl}_{3}\right) \delta$ 20.61, 25.77, 27.11, 31.12, 33.74, 34.50, 34.82, 41.73, 47.76, 60.93, 68.41, 69.01, 112.29, 119.63, 119.81, 130.30, 130.81, 148.33, 152.70, 154.50, 205.10; IR (thin film, $\mathrm{cm}^{-1}$ ) 2932, 2854, 1767, 1721, 1448, 1351, 1240, 1203, 1156; HRMS (ESI) $\mathrm{C}_{21} \mathrm{H}_{30} \mathrm{~N}_{2} \mathrm{O}_{7} \mathrm{SNa}(\mathrm{M}+\mathrm{Na})^{+}$477.1666, found 477.1665 $\mathrm{m} / \mathrm{z}$.

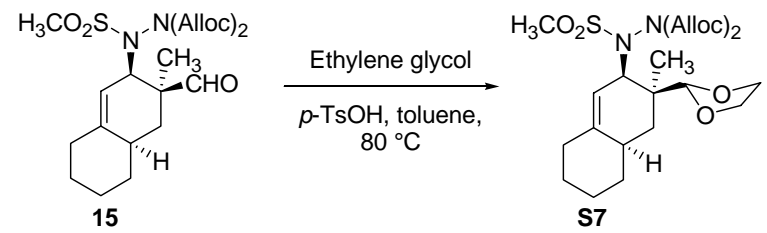

Synthesis of acetal S7: Diels-Alder adduct 15 (120 mg, $0.263 \mathrm{mmol})$, p-toluenesulfonic acid (5 mol\%), and 1:5 toluene/ethylene glycol $(0.25 \mathrm{M})$ were added to a $10 \mathrm{~mL}$ round-bottom flask equipped with a reflux condenser. The reaction was then heated to $80^{\circ} \mathrm{C}$ for $2 \mathrm{~h}$. The reaction mixture was then poured into saturated $\mathrm{NaHCO}_{3}(10 \mathrm{~mL})$ and extracted with methylene chloride (3x20 mL). The combined organics were dried with $\mathrm{Na}_{2} \mathrm{SO}_{4}$ and the solvent was removed by rotary evaporation to provide clear oil. The residue was purified by flash chromatography (3:1 hexanes/ethyl acetate) to provide pure acetal S7 as a clear oil (120 mg, 92\%). ${ }^{1} \mathrm{H}$ NMR (500 MHz, CDCl $) \delta 0.84(\mathrm{~s}, 3 \mathrm{H}), 1.00-1.40(\mathrm{~m}, 4 \mathrm{H}), 1.57-1.64(\mathrm{~m}$, 1H), 1.68-1.79 (m, 3H), 1.86-1.98 (m, 2H), 2.14-2.22 (m, 1H), 3.28 (s, 3H), 3.80-3.94 (m, 4H), 4.25-4.29 (m, 1H), 4.58$4.64(\mathrm{~m}, 1 \mathrm{H}), 4.66-4.79(\mathrm{~m}, 3 \mathrm{H}), 5.34-5.36(\mathrm{~m}, 2 \mathrm{H}), 5.37-5.39(\mathrm{~m}, 1 \mathrm{H}), 5.36-5.48(\mathrm{~m}, 3 \mathrm{H}), 5.85-5.98(\mathrm{~m}, 2 \mathrm{H})$; ${ }^{13} \mathrm{C}$ NMR $\left(125 \mathrm{MHz}, \mathrm{CDCl}_{3}\right) \delta 15.95,26.11,26.97,32.59,33.97,34.25,34.91,41.16,42.17,62.05,64.82,65.09,68.49,68.70,105.63$, 114.90, 119.10, 119.46, 130.61, 130.83, 145.87, 153.32, 153.80; IR (thin film, $\mathrm{cm}^{-1}$ ) 2931, 1768, 1749, 1635, 1347, 1320, 1238, 1196; HRMS (ESI) $\mathrm{C}_{23} \mathrm{H}_{34} \mathrm{~N}_{2} \mathrm{O}_{8} \mathrm{~S}(\mathrm{M}+\mathrm{Na})^{+} 521.1928$, found $521.1928 \mathrm{~m} / \mathrm{z}$.

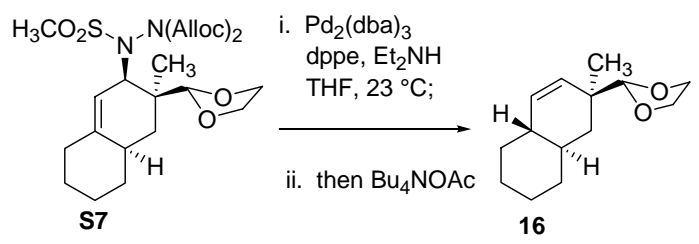

Synthesis of 16: Acetal S7 (120 mg, $0.24 \mathrm{mmol})$ and THF $(1.2 \mathrm{~mL})$ were added to a flame dried, $10 \mathrm{~mL}$ round botton flask under argon. Diethyl amine $(0.375 \mathrm{~mL}, 3.6 \mathrm{mmol})$ was added via syringe. The septa was removed and tris(dibenzylideneacetone)dipalladium chloroform adduct $\left(\mathrm{Pd}_{2}(\mathrm{dba})_{3}\right) \quad(12 \quad \mathrm{mg}, \quad 0.012 \mathrm{mmol})$ and $1,2-$ bis(diphenylphosino)ethane (dppe) $(10 \mathrm{mg}, 0.24 \mathrm{mmol}$ ) were quickly added. The reaction was stirred 30 minutes at ambient temperature or until TLC indicated the absence of starting material at which point tetrabutylammonium acetate was added (288 mg, $0.96 \mathrm{mmol}$ ). The reaction was heated to $35^{\circ} \mathrm{C}$ and stirred $18 \mathrm{~h}$ overnight. The reaction was diluted with a 1:1 mixture of pentane and diethyl ether and the organic layer washed with $\mathrm{H}_{2} \mathrm{O}(2 \mathrm{x})$ and sat. $\mathrm{NaCl}(1 \mathrm{x})$, dried over $\mathrm{Na}_{2} \mathrm{SO}_{4}$, and filtered. The solvent was removed in vacuo and the residual oil purified by column chromatography (95:5 pentane-ether) to yield $37 \mathrm{mg}(70 \%)$ of 16 as a clear oil. ${ }^{1} \mathrm{H}$ NMR (500 MHz, $\left.\mathrm{CDCl}_{3}\right) \delta 1.00(\mathrm{~s}, 3 \mathrm{H}), 0.94-1.08(\mathrm{~m}, 2 \mathrm{H}), 1.13-1.24(\mathrm{~m}, 1 \mathrm{H})$, 
1.25-1.33 (m, 3H), 1.36-1.43 (m, 1H), 1.54-1.62 (m, 2H), 1.66-1.76 (m, 3H), 3.80-3.89 (m, 2H), 3.90-3.96 (m, $2 \mathrm{H}), 4.53$ (s, 1H), 5.39-5.42 (m, 1H), 5.50 (d, $1 \mathrm{H}, \mathrm{J}=10 \mathrm{~Hz}) ;{ }^{13} \mathrm{C}$ NMR (125 MHz, $\left.\mathrm{CDCl}_{3}\right) \delta$ 22.26, 26.70, 26.82, 32.72, 33.40, 36.89, 37.45, 40.93, 42.42, 65.22, 65.33, 109.64, 130.58, 133.27; IR (thin film, $\mathrm{cm}^{-1}$ ) 2921, 2851, 1447, 1116; HRMS (ESI) $\mathrm{C}_{14} \mathrm{H}_{22} \mathrm{O}_{2}(\mathrm{M}+\mathrm{Na})^{+}$245.1512, found $245.1504 \mathrm{~m} / \mathrm{z}$. 
Crystal Structue Data for $\mathbf{8 d}$ :
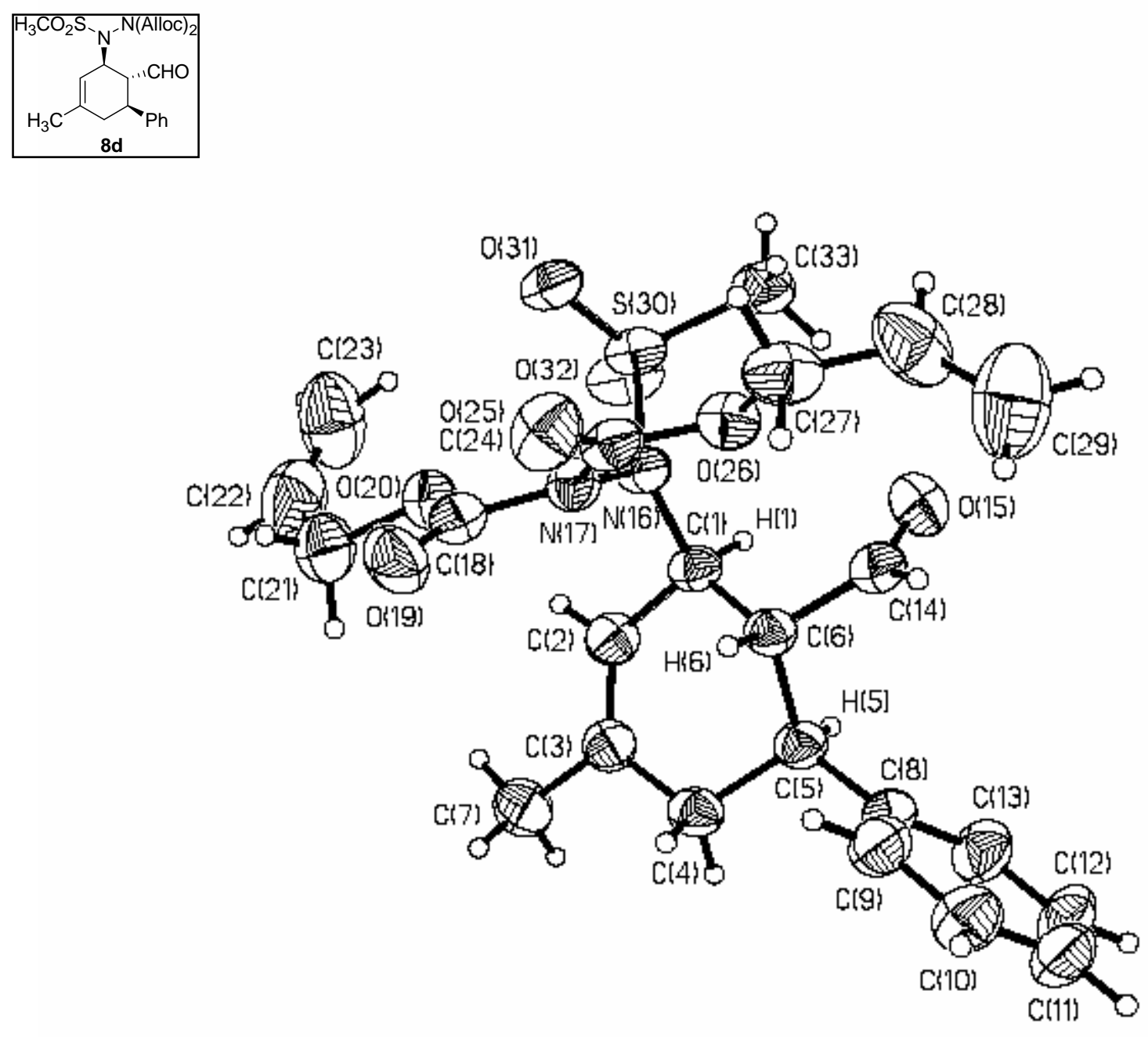
Single-Crystal X-ray Analysis of 8d: Single crystals for X-ray diffraction were obtained from diethyl ether. A colorless needle cut to $0.12 \mathrm{~mm} \times 0.20 \mathrm{~mm} \times 0.33 \mathrm{~mm}$ in size was mounted on a glass fiber with silicone grease and then transferred to a Nonius KappaCCD diffractometer equipped with an MSC X-stream cryosystem and MoK $\alpha$ radiation $(\lambda=0.71073$

Angstroms). Eight hundred frames of data were collected at 200(2) K with an omega oscillation range of $1.0 \mathrm{deg} / \mathrm{frame}$ and an exposure time of $40 \mathrm{sec} /{ }^{\circ}$.(Nonius, 1998) A total of 18,122 reflections (thetamax $=27.56 \mathrm{deg}$ ) were indexed, integrated and corrected for Lorentz and polarization effects using DENZO-SMN and SCALEPACK.(Otwinowski \& Minor, 1997) Data reduction yielded 5646 unique reflections (Rint $=0.048$ ) of which 3986 had $\mathrm{I}>2 \sigma(\mathrm{I})$. Post-refinement of the unit cell parameters gave $\mathrm{a}=9.9186(6) \AA, \mathrm{b}=11.3368(6) \AA, \mathrm{c}=11.9916(6) \AA, \alpha=89.263(2)^{\circ}, \beta=67.540(2)^{\circ}, \gamma=80.873(2)^{\circ}$, and $\mathrm{V}=1228.66(12) \AA^{3}$. Axial photographs and a lack of systematic absences were consistent with the compound having crystallized in the triclinic space group P1 or P-1. The latter centrosymmetric space group P-1 (No. 2) was selected based on an observed mean $\left|E^{*} \mathrm{E}-1\right|$ value of 0.970 (versus the expectation values of 0.968 and 0.736 for centric and noncentric data, respectively). 
Table S1: Fractional atomic coordinates and equivalent isotropic displacement parameters $\left(\AA^{2}\right)$

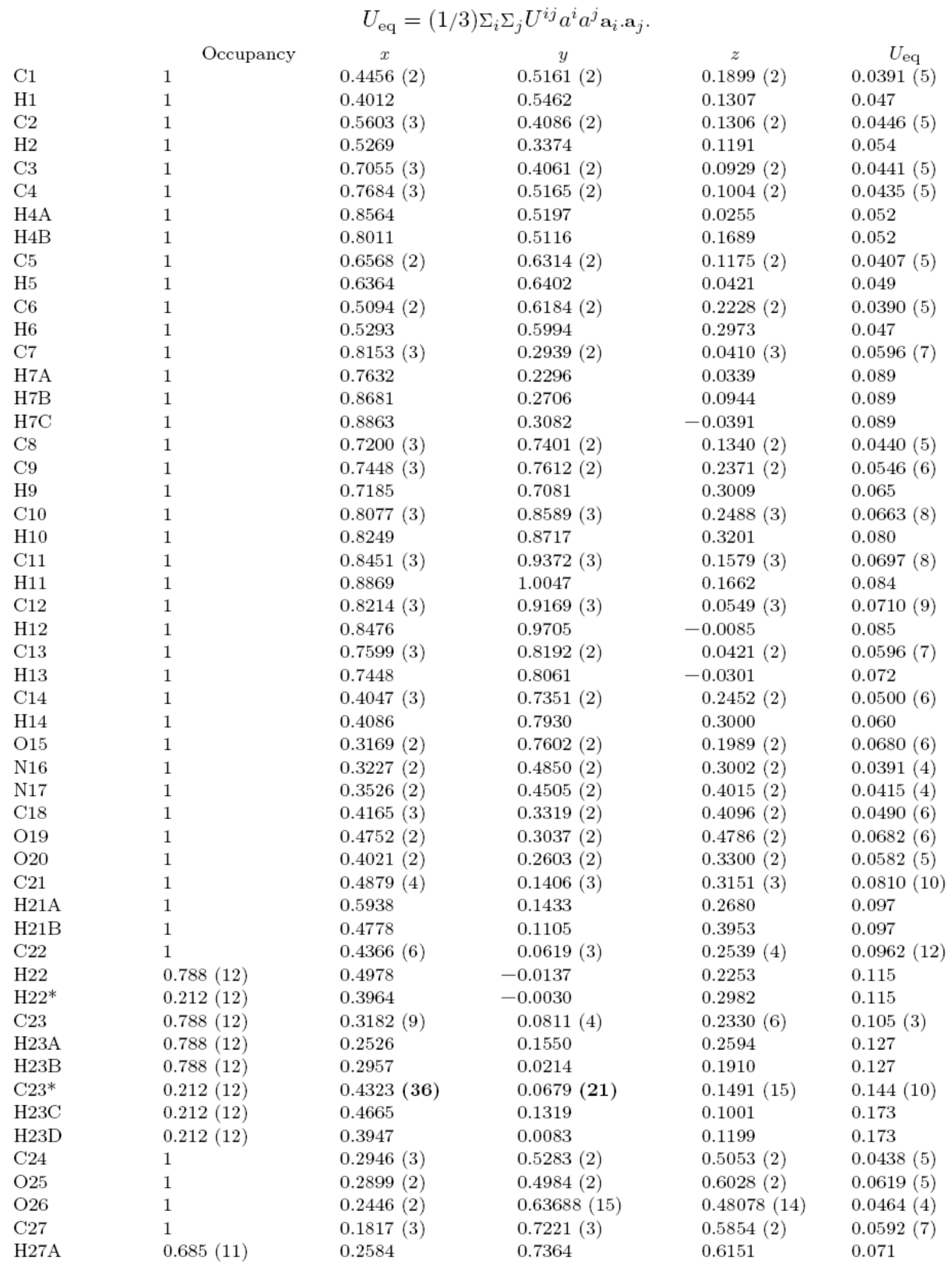




$\begin{array}{llllll}\text { H27B } & 0.685(11) & 0.1010 & 0.6924 & 0.6519 & 0.071 \\ \text { H27C } & 0.315(11) & 0.2486 & 0.7119 & 0.6297 & 0.071 \\ \text { H27D } & 0.315(11) & 0.0862 & 0.7006 & 0.6399 & 0.071 \\ \text { C28 } & 0.685(11) & 0.1239(8) & 0.8331(5) & 0.5398(6) & 0.080(2) \\ \text { H28 } & 0.685(11) & 0.0444 & 0.8317 & 0.5144 & 0.096 \\ \text { C29 } & 0.685(11) & 0.1766(9) & 0.9322(5) & 0.5329(6) & 0.109(3) \\ \text { H29A } & 0.685(11) & 0.2561 & 0.9359 & 0.5577 & 0.130 \\ \text { H29B } & 0.685(11) & 0.1356 & 1.0007 & 0.5030 & 0.130 \\ \text { C28* } & 0.315(11) & 0.1556(16) & 0.8501(8) & 0.5611(17) & 0.095(5) \\ \text { H28* } & 0.315(11) & 0.2330 & 0.8840 & 0.5028 & 0.114 \\ \text { C29* } & 0.315(11) & 0.0284(19) & 0.9166(10) & 0.6183(17) & 0.150(8) \\ \text { H29C } & 0.315(11) & -0.0494 & 0.8833 & 0.6767 & 0.181 \\ \text { H29D } & 0.315(11) & 0.0131 & 0.9988 & 0.6018 & 0.181 \\ \text { S30 } & 1 & 0.16686(6) & 0.45706(6) & 0.29410(5) & 0.0455(2) \\ \text { O31 } & 1 & 0.0979(2) & 0.3947(2) & 0.39964(14) & 0.0506(4) \\ \text { O32 } & 1 & 0.2009(2) & 0.4029(2) & 0.17757(15) & 0.0623(5) \\ \text { C33 } & 1 & 0.0597(3) & 0.5973(3) & 0.3049(2) & 0.0556(7) \\ \text { H33A } & 1 & -0.0310 & 0.5880 & 0.2938 & 0.083 \\ \text { H33B } & 1 & 0.1159 & 0.6470 & 0.2423 & 0.083 \\ \text { H33C } & 1 & 0.0339 & 0.6357 & 0.3848 & 0.083\end{array}$

Table S2: Anisotropic displacement parameters $\left(\AA^{2}\right)$

\begin{tabular}{|c|c|c|c|c|c|c|}
\hline & $U_{11}$ & $U_{22}$ & $U_{33}$ & $U_{12}$ & $U_{13}$ & $U_{23}$ \\
\hline $\mathrm{C} 1$ & $0.0339(12)$ & $0.0474(13)$ & $0.0343(11)$ & $-0.0097(10)$ & $-0.0103(9)$ & $0.0048(9)$ \\
\hline $\mathrm{C} 2$ & $0.0449(14)$ & 0.0439 (13) & $0.0414(12)$ & $-0.0069(10)$ & $-0.0126(10)$ & $-0.0023(10)$ \\
\hline C3 & $0.0421(14)$ & $0.0476(14)$ & $0.0375(12)$ & $-0.0054(10)$ & $-0.0107(10)$ & $0.0036(10)$ \\
\hline $\mathrm{C} 4$ & $0.0354(12)$ & 0.0518 (14) & $0.0383(12)$ & $-0.0050(10)$ & $-0.0093(10)$ & $0.0031(10)$ \\
\hline C5 & $0.0357(12)$ & $0.0473(13)$ & $0.0369(11)$ & $-0.0085(10)$ & $-0.0113(9)$ & $0.0080(10)$ \\
\hline C6 & $0.0336(12)$ & 0.0438 (13) & 0.0358 (11) & $-0.0061(9)$ & $-0.0093(9)$ & $0.0046(9)$ \\
\hline $\mathrm{C} 7$ & $0.051(2)$ & $0.054(2)$ & $0.060(2)$ & $0.0006(13)$ & $-0.0109(13)$ & -0.0037 \\
\hline $\mathrm{C} 8$ & $0.0338(12)$ & 0.0447 (13) & $0.0463(13)$ & $-0.0070(10)$ & $-0.0073(10)$ & $0.0071(10)$ \\
\hline C9 & $0.053(2)$ & $0.056(2)$ & $0.060(2)$ & $-0.0173(13)$ & $-0.0251(13)$ & $0.0116(13)$ \\
\hline $\mathrm{C} 10$ & $0.063(2)$ & $0.061(2)$ & $0.081(2)$ & $-0.0183(15)$ & $-0.031(2)$ & $-0.001(2)$ \\
\hline $\mathrm{C} 11$ & $0.056(2)$ & $0.052(2)$ & $0.091(2)$ & $-0.0205(14)$ & $-0.013(2)$ & $-0.002(2)$ \\
\hline $\mathrm{C} 12$ & $0.067(2)$ & $0.051(2)$ & $0.077(2)$ & $-0.0224(15)$ & $-0.003(2)$ & 0.0143 \\
\hline $\mathrm{C} 13$ & $0.059(2)$ & $0.058(2)$ & $0.0518(15)$ & $-0.0162(13)$ & $-0.0079(13)$ & 0.0117 \\
\hline $\mathrm{C} 14$ & $0.0390(14)$ & 0.0464 (14) & $0.0555(15)$ & $-0.0100(11)$ & $-0.0072(12)$ & 0.0052 \\
\hline O15 & $0.0573(13)$ & $0.0581(12)$ & $0.0896(15)$ & $-0.0008(9)$ & $-0.0329(11)$ & $0.0129(11)$ \\
\hline N16 & $0.0343(10)$ & $0.0498(11)$ & $0.0349(9)$ & $-0.0109(8)$ & $-0.0138(8)$ & $0.0063(8)$ \\
\hline N17 & $0.0403(11)$ & 0.0484 (11) & $0.0382(10)$ & $-0.0104(9)$ & $-0.0167(8)$ & $0.0113(8)$ \\
\hline $\mathrm{C} 18$ & $0.0388(13)$ & $0.0529(15)$ & 0.0491 (14) & $-0.0094(11)$ & $-0.0095(11)$ & $0.0111(12)$ \\
\hline O19 & $0.0585(12)$ & $0.0802(14)$ & $0.0640(12)$ & $0.0020(10)$ & $-0.0271(10)$ & $0.0216(10)$ \\
\hline $\mathrm{O} 20$ & $0.0531(11)$ & $0.0423(10)$ & $0.0744(12)$ & $-0.0062(8)$ & $-0.0199(9)$ & $0.0059(9)$ \\
\hline $\mathrm{C} 21$ & $0.072(2)$ & $0.045(2)$ & $0.109(3)$ & $-0.0026(15)$ & $-0.019(2)$ & $0.005(2)$ \\
\hline $\mathrm{C} 22$ & 0.118 (4) & $0.058(2)$ & $0.103(3)$ & $-0.017(2)$ & -0.031 & $0.003(2)$ \\
\hline $\mathrm{C} 23$ & $0.154(7)$ & $0.063(3)$ & $0.134(6)$ & $-0.018(3)$ & $-0.093(6)$ & $0.002(3)$ \\
\hline $\mathrm{C} 24$ & $0.0392(13)$ & $0.0574(15)$ & $0.0403(12)$ & $-0.0180(11)$ & $-0.0175(10)$ & $0.0101(11)$ \\
\hline $\mathrm{O} 25$ & $0.0784(14)$ & $0.0730(13)$ & $0.0448(10)$ & $-0.0223(11)$ & $-0.0317(10)$ & $0.0144(9)$ \\
\hline $\mathrm{O} 26$ & $0.0441(10)$ & $0.0536(10)$ & $0.0413(9)$ & $-0.0077(8)$ & $-0.0163(7)$ & $0.0019(7)$ \\
\hline $\mathrm{C} 27$ & $0.049(2)$ & $0.071(2)$ & $0.0543(15)$ & $-0.0124(13)$ & $-0.0137(12)$ & -0.0141 \\
\hline $\mathrm{C} 28$ & $0.079(4)$ & $0.063(3)$ & $0.098(4)$ & $0.011(3)$ & $-0.043(3)$ & $-0.032(3)$ \\
\hline $\mathrm{C} 29$ & $0.165(8)$ & $0.070(4)$ & $0.097(5)$ & $-0.023(4)$ & $-0.056(5)$ & $-0.004(3)$ \\
\hline S30 & $0.0373(3)$ & 0.0617 (4) & $0.0385(3)$ & -0.0147 & $-0.0131(3)$ & $0.0030(3)$ \\
\hline O31 & $0.0408(10)$ & $0.0627(11)$ & $0.0467(9)$ & -0.0190 & $-0.0115(8)$ & $0.0096(8)$ \\
\hline $\mathrm{O} 32$ & $0.0533(11)$ & $0.0951(15)$ & $0.0413(9)$ & $-0.0256(10)$ & -0.0156 & $-0.0079(9)$ \\
\hline C33 & 0.0401 (14) & $0.075(2)$ & $0.0555(15)$ & $-0.0093(12)$ & $-0.0228(12)$ & 0.0190 \\
\hline
\end{tabular}


Table S3: Geometric parameters $\left(\AA^{2},{ }^{\circ}\right)$

\begin{tabular}{|c|c|}
\hline $\mathrm{C} 1-\mathrm{C} 2$ & 1.499 (3) \\
\hline $\mathrm{C} 1-\mathrm{N} 16$ & $1.500(3)$ \\
\hline $\mathrm{C} 1-\mathrm{C} 6$ & $1.529(3)$ \\
\hline C1-H1 & 1.00 \\
\hline $\mathrm{C} 2-\mathrm{C} 3$ & $1.331(3)$ \\
\hline $\mathrm{C} 2-\mathrm{H} 2$ & 0.95 \\
\hline $\mathrm{C} 3-\mathrm{C} 7$ & $1.502(3)$ \\
\hline C3-C4 & $1.502(3)$ \\
\hline $\mathrm{C} 4-\mathrm{C} 5$ & $1.530(3)$ \\
\hline $\mathrm{C} 4-\mathrm{H} 4 \mathrm{~A}$ & 0.99 \\
\hline $\mathrm{C} 4-\mathrm{H} 4 \mathrm{~B}$ & 0.99 \\
\hline C5-C8 & $1.515(3)$ \\
\hline C5-C6 & 1.551 (3) \\
\hline C5-H5 & 1.00 \\
\hline C6-C14 & $1.503(3)$ \\
\hline C6-H6 & 1.00 \\
\hline C7-H7A & 0.98 \\
\hline C7-H7B & 0.98 \\
\hline $\mathrm{C} 7-\mathrm{H} 7 \mathrm{C}$ & 0.98 \\
\hline C8-C9 & $1.381(3)$ \\
\hline $\mathrm{C} 8-\mathrm{C} 13$ & $1.389(3)$ \\
\hline $\mathrm{C} 9-\mathrm{C} 10$ & $1.388(4)$ \\
\hline C9-H9 & 0.95 \\
\hline $\mathrm{C} 10-\mathrm{C} 11$ & $1.373(4)$ \\
\hline C10-H10 & 0.95 \\
\hline $\mathrm{C} 11-\mathrm{C} 12$ & $1.371(4)$ \\
\hline C11-H11 & 0.95 \\
\hline $\mathrm{C} 12-\mathrm{C} 13$ & $1.383(4)$ \\
\hline C12-H12 & 0.95 \\
\hline C13-H13 & 0.95 \\
\hline C14-O15 & $1.198(3)$ \\
\hline C14-H14 & 0.95 \\
\hline N16-N17 & $1.395(2)$ \\
\hline N16-S30 & $1.654(2)$ \\
\hline $\mathrm{N} 17-\mathrm{C} 24$ & 1.407 (3) \\
\hline N17-C18 & $1.411(3)$ \\
\hline C18-O19 & $1.195(3)$ \\
\hline
\end{tabular}

$\begin{array}{ll}\mathrm{C} 18-\mathrm{O} 20 & 1.324(3) \\ \mathrm{O} 20-\mathrm{C} 21 & 1.456(3) \\ \mathrm{C} 21-\mathrm{C} 22 & 1.429(5) \\ \mathrm{C} 21-\mathrm{H} 21 \mathrm{~A} & 0.99 \\ \mathrm{C} 21-\mathrm{H} 21 \mathrm{~B} & 0.99 \\ \mathrm{C} 22-\mathrm{C} 23^{*} & 1.274(7) \\ \mathrm{C} 22-\mathrm{C} 23 & 1.277(7) \\ \mathrm{C} 22-\mathrm{H} 22 & 0.95 \\ \mathrm{C} 22-\mathrm{H} 22^{*} & 0.95 \\ \mathrm{C} 23-\mathrm{H} 23 \mathrm{~A} & 0.95 \\ \mathrm{C} 23-\mathrm{H} 23 \mathrm{~B} & 0.95 \\ \mathrm{C} 23^{*}-\mathrm{H} 23 \mathrm{C} & 0.95 \\ \mathrm{C} 23^{*}-\mathrm{H} 23 \mathrm{D} & 0.95 \\ \mathrm{C} 24-\mathrm{O} 25 & 1.199(3) \\ \mathrm{C} 24-\mathrm{O} 26 & 1.325(3) \\ \mathrm{O} 26-\mathrm{C} 27 & 1.463(3) \\ \mathrm{C} 27-\mathrm{C} 28^{*} & 1.478(7) \\ \mathrm{C} 27-\mathrm{C} 28 & 1.484(6) \\ \mathrm{C} 27-\mathrm{H} 27 \mathrm{~A} & 0.99 \\ \mathrm{C} 27-\mathrm{H} 27 \mathrm{~B} & 0.99 \\ \mathrm{C} 27-\mathrm{H} 27 \mathrm{C} & 0.99 \\ \mathrm{C} 27-\mathrm{H} 27 \mathrm{D} & 0.99 \\ \mathrm{C} 28-\mathrm{C} 29 & 1.301(10) \\ \mathrm{C} 28-\mathrm{H} 28 & 0.95 \\ \mathrm{C} 29-\mathrm{H} 29 \mathrm{~A} & 0.95 \\ \mathrm{C} 29-\mathrm{H} 29 \mathrm{~B} & 0.95 \\ \mathrm{C} 28^{*}-\mathrm{C} 29^{*} & 1.297(7) \\ \mathrm{C} 28^{*}-\mathrm{H} 2{ }^{*} & 0.95 \\ \mathrm{C} 29^{*}-\mathrm{H} 29 \mathrm{C} & 0.95 \\ \mathrm{C} 29^{*}-\mathrm{H} 29 \mathrm{D} & 0.95 \\ \mathrm{~S} 30-\mathrm{O} 31 & 1.428(2) \\ \mathrm{S} 30-\mathrm{O} 32 & 1.428(2) \\ \mathrm{S} 30-\mathrm{C} 33 & 1.743(3) \\ \mathrm{C} 33-\mathrm{H} 33 \mathrm{~A} & 0.98 \\ \mathrm{C} 33-\mathrm{H} 33 \mathrm{~B} & 0.98 \\ \mathrm{C} 33-\mathrm{H} 33 \mathrm{C} & 0.98 \\ & \end{array}$




\begin{tabular}{|c|c|c|c|}
\hline $\mathrm{C} 2-\mathrm{C} 1-\mathrm{N} 16$ & $111.9(2)$ & $\mathrm{O} 19-\mathrm{C} 18-\mathrm{O} 20$ & $126.6(2)$ \\
\hline $\mathrm{C} 2-\mathrm{C} 1-\mathrm{C} 6$ & $113.2(2)$ & O19-C18-N17 & $123.5(3)$ \\
\hline $\mathrm{N} 16-\mathrm{C} 1-\mathrm{C} 6$ & $109.5(2)$ & $\mathrm{O} 20-\mathrm{C} 18-\mathrm{N} 17$ & $109.9(2)$ \\
\hline $\mathrm{C} 2-\mathrm{C} 1-\mathrm{H} 1$ & 107.3 & $\mathrm{C} 18-\mathrm{O} 20-\mathrm{C} 21$ & $114.2(2)$ \\
\hline $\mathrm{N} 16-\mathrm{C} 1-\mathrm{H} 1$ & 107.3 & $\mathrm{C} 22-\mathrm{C} 21-\mathrm{O} 20$ & $109.9(3)$ \\
\hline C6-C1-H1 & 107.3 & $\mathrm{C} 22-\mathrm{C} 21-\mathrm{H} 21 \mathrm{~A}$ & 109.7 \\
\hline $\mathrm{C} 3-\mathrm{C} 2-\mathrm{C} 1$ & $124.5(2)$ & $\mathrm{O} 20-\mathrm{C} 21-\mathrm{H} 21 \mathrm{~A}$ & 109.7 \\
\hline $\mathrm{C} 3-\mathrm{C} 2-\mathrm{H} 2$ & 117.7 & C22-C21-H21B & 109.7 \\
\hline $\mathrm{C} 1-\mathrm{C} 2-\mathrm{H} 2$ & 117.7 & $\mathrm{O} 20-\mathrm{C} 21-\mathrm{H} 21 \mathrm{~B}$ & 109.7 \\
\hline $\mathrm{C} 2-\mathrm{C} 3-\mathrm{C} 7$ & $122.3(2)$ & $\mathrm{H} 21 \mathrm{~A}-\mathrm{C} 21-\mathrm{H} 21 \mathrm{~B}$ & 108.2 \\
\hline $\mathrm{C} 2-\mathrm{C} 3-\mathrm{C} 4$ & $121.4(2)$ & $\mathrm{C} 23^{*}-\mathrm{C} 22-\mathrm{C} 21$ & $129.4(9)$ \\
\hline $\mathrm{C} 7-\mathrm{C} 3-\mathrm{C} 4$ & $116.3(2)$ & $\mathrm{C} 23-\mathrm{C} 22-\mathrm{C} 21$ & $127.7(4)$ \\
\hline $\mathrm{C} 3-\mathrm{C} 4-\mathrm{C} 5$ & $112.5(2)$ & $\mathrm{C} 23-\mathrm{C} 22-\mathrm{H} 22$ & 116.2 \\
\hline $\mathrm{C} 3-\mathrm{C} 4-\mathrm{H} 4 \mathrm{~A}$ & 109.1 & $\mathrm{C} 21-\mathrm{C} 22-\mathrm{H} 22$ & 116.2 \\
\hline $\mathrm{C} 5-\mathrm{C} 4-\mathrm{H} 4 \mathrm{~A}$ & 109.1 & $\mathrm{C} 23^{*}-\mathrm{C} 22-\mathrm{H} 22^{*}$ & 114.6 \\
\hline C3-C4-H4B & 109.1 & C21-C22-H22* & 115.9 \\
\hline $\mathrm{C} 5-\mathrm{C} 4-\mathrm{H} 4 \mathrm{~B}$ & 109.1 & $\mathrm{C} 22-\mathrm{C} 23-\mathrm{H} 23 \mathrm{~A}$ & 120.0 \\
\hline $\mathrm{H} 4 \mathrm{~A}-\mathrm{C} 4-\mathrm{H} 4 \mathrm{~B}$ & 107.8 & $\mathrm{C} 22-\mathrm{C} 23-\mathrm{H} 23 \mathrm{~B}$ & 120.0 \\
\hline $\mathrm{C} 8-\mathrm{C} 5-\mathrm{C} 4$ & $111.1(2)$ & $\mathrm{H} 23 \mathrm{~A}-\mathrm{C} 23-\mathrm{H} 23 \mathrm{~B}$ & 120.0 \\
\hline $\mathrm{C} 8-\mathrm{C} 5-\mathrm{C} 6$ & $113.5(2)$ & $\mathrm{C} 22-\mathrm{C} 23^{*}-\mathrm{H} 23 \mathrm{C}$ & 120.0 \\
\hline $\mathrm{C} 4-\mathrm{C} 5-\mathrm{C} 6$ & $109.2(2)$ & $\mathrm{C} 22-\mathrm{C} 23 *-\mathrm{H} 23 \mathrm{D}$ & 120.0 \\
\hline C8-C5-H5 & 107.6 & $\mathrm{H} 23 \mathrm{C}-\mathrm{C} 23^{*}-\mathrm{H} 23 \mathrm{D}$ & 120.0 \\
\hline C4-C5-H5 & 107.6 & $\mathrm{O} 25-\mathrm{C} 24-\mathrm{O} 26$ & $125.3(2)$ \\
\hline C6-C5-H5 & 107.6 & $\mathrm{O} 25-\mathrm{C} 24-\mathrm{N} 17$ & $123.7(2)$ \\
\hline C14-C6-C1 & $111.6(2)$ & O26-C24-N17 & $111.0(2)$ \\
\hline C14-C6-C5 & $108.8(2)$ & $\mathrm{C} 24-\mathrm{O} 26-\mathrm{C} 27$ & $113.7(2)$ \\
\hline $\mathrm{C} 1-\mathrm{C} 6-\mathrm{C} 5$ & $109.2(2)$ & $\mathrm{O} 26-\mathrm{C} 27-\mathrm{C} 28^{*}$ & $116.9(8)$ \\
\hline C14-C6-H6 & 109.1 & $\mathrm{O} 26-\mathrm{C} 27-\mathrm{C} 28$ & $104.5(3)$ \\
\hline $\mathrm{C} 1-\mathrm{C} 6-\mathrm{H} 6$ & 109.1 & $\mathrm{O} 26-\mathrm{C} 27-\mathrm{H} 27 \mathrm{~A}$ & 110.8 \\
\hline $\mathrm{C} 5-\mathrm{C} 6-\mathrm{H} 6$ & 109.1 & $\mathrm{C} 28-\mathrm{C} 27-\mathrm{H} 27 \mathrm{~A}$ & 110.8 \\
\hline C3-C7-H7A & 109.5 & $\mathrm{O} 26-\mathrm{C} 27-\mathrm{H} 27 \mathrm{~B}$ & 110.8 \\
\hline $\mathrm{C} 3-\mathrm{C} 7-\mathrm{H} 7 \mathrm{~B}$ & 109.5 & $\mathrm{C} 28-\mathrm{C} 27-\mathrm{H} 27 \mathrm{~B}$ & 110.8 \\
\hline $\mathrm{H} 7 \mathrm{~A}-\mathrm{C} 7-\mathrm{H} 7 \mathrm{~B}$ & 109.5 & $\mathrm{H} 27 \mathrm{~A}-\mathrm{C} 27-\mathrm{H} 27 \mathrm{~B}$ & 108.9 \\
\hline C3-C7-H7C & 109.5 & $\mathrm{O} 26-\mathrm{C} 27-\mathrm{H} 27 \mathrm{C}$ & 108.1 \\
\hline $\mathrm{H} 7 \mathrm{~A}-\mathrm{C} 7-\mathrm{H} 7 \mathrm{C}$ & 109.5 & $\mathrm{C} 28^{*}-\mathrm{C} 27-\mathrm{H} 27 \mathrm{C}$ & 108.1 \\
\hline $\mathrm{H} 7 \mathrm{~B}-\mathrm{C} 7-\mathrm{H} 7 \mathrm{C}$ & 109.5 & $\mathrm{O} 26-\mathrm{C} 27-\mathrm{H} 27 \mathrm{D}$ & 108.1 \\
\hline $\mathrm{C} 9-\mathrm{C} 8-\mathrm{C} 13$ & $118.2(2)$ & $\mathrm{C} 28^{*}-\mathrm{C} 27-\mathrm{H} 27 \mathrm{D}$ & 108.1 \\
\hline C9-C8-C5 & $121.7(2)$ & $\mathrm{H} 27 \mathrm{C}-\mathrm{C} 27-\mathrm{H} 27 \mathrm{D}$ & 107.3 \\
\hline $\mathrm{C} 13-\mathrm{C} 8-\mathrm{C} 5$ & $120.0(2)$ & $\mathrm{C} 29-\mathrm{C} 28-\mathrm{C} 27$ & $123.3(7)$ \\
\hline C8-C9-C10 & $121.0(3)$ & $\mathrm{C} 29-\mathrm{C} 28-\mathrm{H} 28$ & 118.3 \\
\hline C8-C9-H9 & 119.5 & C27-C28-H28 & 118.3 \\
\hline C10-C9-H9 & 119.5 & C28-C29-H29A & 120.0 \\
\hline C11-C10-C9 & $120.2(3)$ & C28-C29-H29B & 120.0 \\
\hline $\mathrm{C} 11-\mathrm{C} 10-\mathrm{H} 10$ & 119.9 & H29A-C29-H29B & 120.0 \\
\hline $\mathrm{C} 9-\mathrm{C} 10-\mathrm{H} 10$ & 119.9 & $\mathrm{C} 29^{*}-\mathrm{C} 28^{*}-\mathrm{C} 27$ & $120.8(9)$ \\
\hline $\mathrm{C} 12-\mathrm{C} 11-\mathrm{C} 10$ & $119.3(3)$ & $\mathrm{C} 29^{*}-\mathrm{C} 28^{*}-\mathrm{H} 28^{*}$ & 119.6 \\
\hline C12-C11-H11 & 120.3 & $\mathrm{C} 27-\mathrm{C} 28^{*}-\mathrm{H} 28^{*}$ & 119.6 \\
\hline C10-C11-H11 & 120.3 & $\mathrm{C} 28^{*}-\mathrm{C} 29^{*}-\mathrm{H} 29 \mathrm{C}$ & 120.0 \\
\hline $\mathrm{C} 11-\mathrm{C} 12-\mathrm{C} 13$ & $120.8(3)$ & $\mathrm{C} 28^{*}-\mathrm{C} 29^{*}-\mathrm{H} 29 \mathrm{D}$ & 120.0 \\
\hline $\mathrm{C} 11-\mathrm{C} 12-\mathrm{H} 12$ & 119.6 & $\mathrm{H} 29 \mathrm{C}-\mathrm{C} 29^{*}-\mathrm{H} 29 \mathrm{D}$ & 120.0 \\
\hline $\mathrm{C} 13-\mathrm{C} 12-\mathrm{H} 12$ & 119.6 & $\mathrm{O} 31-\mathrm{S} 30-\mathrm{O} 32$ & $119.55(11)$ \\
\hline $\mathrm{C} 12-\mathrm{C} 13-\mathrm{C} 8$ & $120.5(3)$ & O31-S30-N16 & $106.34(9)$ \\
\hline C12-C13-H13 & 119.8 & O32-S30-N16 & $108.00(10)$ \\
\hline C8-C13-H13 & 119.8 & $\mathrm{O} 31-\mathrm{S} 30-\mathrm{C} 33$ & $108.95(12)$ \\
\hline $\mathrm{O} 15-\mathrm{C} 14-\mathrm{C} 6$ & $124.7(2)$ & O32-S30-C33 & $108.10(13)$ \\
\hline O15-C14-H14 & 117.7 & N16-S30-C33 & $104.95(11)$ \\
\hline C6-C14-H14 & 117.7 & S30-C33-H33A & 109.5 \\
\hline N17-N16-C1 & $119.0(2)$ & S30-C33-H33B & 109.5 \\
\hline N17-N16-S30 & $117.71(14)$ & H33A-C $33-$ H33B & 109.5 \\
\hline C1-N16-S30 & $121.47(14)$ & S30-C33-H33C & 109.5 \\
\hline $\mathrm{N} 16-\mathrm{N} 17-\mathrm{C} 24$ & $119.2(2)$ & H33A-C33-H33C & 109.5 \\
\hline N16-N17-C18 & $121.0(2)$ & Н33В-С $33-$ Н33C & 109.5 \\
\hline C24-N17-C18 & $118.9(2)$ & & \\
\hline
\end{tabular}




\begin{tabular}{|c|c|c|c|}
\hline $\mathrm{N} 16-\mathrm{C} 1-\mathrm{C} 2-\mathrm{C} 3$ & $-133.9(2)$ & C6-C1-N16-S30 & $137.7(2)$ \\
\hline $\mathrm{C} 6-\mathrm{C} 1-\mathrm{C} 2-\mathrm{C} 3$ & $-9.6(3)$ & $\mathrm{C} 1-\mathrm{N} 16-\mathrm{N} 17-\mathrm{C} 24$ & $109.5(2)$ \\
\hline $\mathrm{C} 1-\mathrm{C} 2-\mathrm{C} 3-\mathrm{C} 7$ & $176.0(2)$ & S30-N16-N17-C24 & $-85.7(2)$ \\
\hline $\mathrm{C} 1-\mathrm{C} 2-\mathrm{C} 3-\mathrm{C} 4$ & $-3.8(3)$ & C1-N16-N17-C18 & $-81.9(3)$ \\
\hline $\mathrm{C} 2-\mathrm{C} 3-\mathrm{C} 4-\mathrm{C} 5$ & $-17.3(3)$ & S30-N16-N17-C18 & $82.8(2)$ \\
\hline $\mathrm{C} 7-\mathrm{C} 3-\mathrm{C} 4-\mathrm{C} 5$ & $162.8(2)$ & N16-N17-C18-O19 & $162.1(2)$ \\
\hline $\mathrm{C} 3-\mathrm{C} 4-\mathrm{C} 5-\mathrm{C} 8$ & $175.8(2)$ & C24-N17-C18-O19 & $-29.3(3)$ \\
\hline C3-C4-C5-C6 & $49.8(2)$ & N16-N17-C18-O20 & $-17.3(3)$ \\
\hline $\mathrm{C} 2-\mathrm{C} 1-\mathrm{C} 6-\mathrm{C} 14$ & $162.2(2)$ & $\mathrm{C} 24-\mathrm{N} 17-\mathrm{C} 18-\mathrm{O} 20$ & $151.2(2)$ \\
\hline $\mathrm{N} 16-\mathrm{C} 1-\mathrm{C} 6-\mathrm{C} 14$ & $-72.2(2)$ & $\mathrm{O} 19-\mathrm{C} 18-\mathrm{O} 20-\mathrm{C} 21$ & $-10.3(4)$ \\
\hline $\mathrm{C} 2-\mathrm{C} 1-\mathrm{C} 6-\mathrm{C} 5$ & $42.0(2)$ & $\mathrm{N} 17-\mathrm{C} 18-\mathrm{O} 20-\mathrm{C} 21$ & $169.2(2)$ \\
\hline $\mathrm{N} 16-\mathrm{C} 1-\mathrm{C} 6-\mathrm{C} 5$ & $167.6(2)$ & $\mathrm{C} 18-\mathrm{O} 20-\mathrm{C} 21-\mathrm{C} 22$ & $164.7(3)$ \\
\hline $\mathrm{C} 8-\mathrm{C} 5-\mathrm{C} 6-\mathrm{C} 14$ & $50.9(3)$ & O $20-\mathrm{C} 21-\mathrm{C} 22-\mathrm{C} 23^{*}$ & $60.1(21)$ \\
\hline $\mathrm{C} 4-\mathrm{C} 5-\mathrm{C} 6-\mathrm{C} 14$ & $175.6(2)$ & $\mathrm{O} 20-\mathrm{C} 21-\mathrm{C} 22-\mathrm{C} 23$ & $-12.4(7)$ \\
\hline $\mathrm{C} 8-\mathrm{C} 5-\mathrm{C} 6-\mathrm{C} 1$ & $172.9(2)$ & $\mathrm{N} 16-\mathrm{N} 17-\mathrm{C} 24-\mathrm{O} 25$ & $165.3(2)$ \\
\hline $\mathrm{C} 4-\mathrm{C} 5-\mathrm{C} 6-\mathrm{C} 1$ & $-62.5(2)$ & $\mathrm{C} 18-\mathrm{N} 17-\mathrm{C} 24-\mathrm{O} 25$ & $-3.5(3)$ \\
\hline $\mathrm{C} 4-\mathrm{C} 5-\mathrm{C} 8-\mathrm{C} 9$ & $-69.7(3)$ & $\mathrm{N} 16-\mathrm{N} 17-\mathrm{C} 24-\mathrm{O} 26$ & $-13.8(3)$ \\
\hline C6-C5-C8-C9 & $53.9(3)$ & $\mathrm{C} 18-\mathrm{N} 17-\mathrm{C} 24-\mathrm{O} 26$ & $177.4(2)$ \\
\hline $\mathrm{C} 4-\mathrm{C} 5-\mathrm{C} 8-\mathrm{C} 13$ & $107.9(2)$ & $\mathrm{O} 25-\mathrm{C} 24-\mathrm{O} 26-\mathrm{C} 27$ & $0.5(3)$ \\
\hline $\mathrm{C} 6-\mathrm{C} 5-\mathrm{C} 8-\mathrm{C} 13$ & $-128.5(2)$ & $\mathrm{N} 17-\mathrm{C} 24-\mathrm{O} 26-\mathrm{C} 27$ & $179.6(2)$ \\
\hline C13-C8-C9-C10 & $0.1(4)$ & $\mathrm{C} 24-\mathrm{O} 26-\mathrm{C} 27-\mathrm{C} 28^{*}$ & $166.2(7)$ \\
\hline $\mathrm{C} 5-\mathrm{C} 8-\mathrm{C} 9-\mathrm{C} 10$ & $177.7(2)$ & $\mathrm{C} 24-\mathrm{O} 26-\mathrm{C} 27-\mathrm{C} 28$ & $-176.1(3)$ \\
\hline C8-C9-C10-C11 & $0.6(5)$ & $\mathrm{O} 26-\mathrm{C} 27-\mathrm{C} 28-\mathrm{C} 29$ & $-114.9(6)$ \\
\hline C9-C10-C11-C12 & $-0.8(5)$ & $\mathrm{C} 28^{*}-\mathrm{C} 27-\mathrm{C} 28-\mathrm{C} 29$ & $14.8(19)$ \\
\hline $\mathrm{C} 10-\mathrm{C} 11-\mathrm{C} 12-\mathrm{C} 13$ & $0.3(5)$ & $\mathrm{O} 26-\mathrm{C} 27-\mathrm{C} 28 *-\mathrm{C} 29 *$ & $129.4(17)$ \\
\hline $\mathrm{C} 11-\mathrm{C} 12-\mathrm{C} 13-\mathrm{C} 8$ & $0.4(5)$ & $\mathrm{C} 28-\mathrm{C} 27-\mathrm{C} 28^{*}-\mathrm{C} 29^{*}$ & $72.8(21)$ \\
\hline C9-C8-C13-C12 & -0.6 & N17-N16-S30-O31 & $-2.7(2)$ \\
\hline $\mathrm{C} 5-\mathrm{C} 8-\mathrm{C} 13-\mathrm{C} 12$ & $-178.2(3)$ & C1-N16-S30-O31 & $161.6(2)$ \\
\hline $\mathrm{C} 1-\mathrm{C} 6-\mathrm{C} 14-\mathrm{O} 15$ & $-28.9(3)$ & N17-N16-S30-O32 & $-132.2(2)$ \\
\hline C5-C6-C14-O15 & $91.6(3)$ & $\mathrm{C} 1-\mathrm{N} 16-\mathrm{S} 30-\mathrm{O} 32$ & $32.2(2)$ \\
\hline $\mathrm{C} 2-\mathrm{C} 1-\mathrm{N} 16-\mathrm{N} 17$ & $68.2(2)$ & N17-N16-S30-C33 & $112.7(2)$ \\
\hline C6-C1-N16-N17 & $-58.2(2)$ & C1-N16-S30-C33 & $-83.0(2)$ \\
\hline $\mathrm{C} 2-\mathrm{C} 1-\mathrm{N} 16-\mathrm{S} 30$ & $-96.0(2)$ & & \\
\hline
\end{tabular}

